$1-1-2009$

\title{
Characterization and analysis of edges using the continuous shearlet transform
}

Kanghui Guo

Missouri State University

Demetrio Labate

Follow this and additional works at: https://bearworks.missouristate.edu/articles-cnas

\section{Recommended Citation}

Guo, Kanghui, and Demetrio Labate. "Characterization and analysis of edges using the continuous shearlet transform." SIAM journal on Imaging Sciences 2, no. 3 (2009): 959-986.

This article or document was made available through BearWorks, the institutional repository of Missouri State University. The work contained in it may be protected by copyright and require permission of the copyright holder for reuse or redistribution.

For more information, please contact BearWorks@library.missouristate.edu. 


\title{
CHARACTERIZATION AND ANALYSIS OF EDGES USING THE CONTINUOUS SHEARLET TRANSFORM
}

\author{
KANGHUI GUO* AND DEMETRIO LABATE ${ }^{\dagger}$
}

\begin{abstract}
This paper shows that the continuous shearlet transform, a novel directional multiscale transform recently introduced by the authors and their collaborators, provides a precise geometrical characterization for the boundary curves of very general planar regions. This study is motivated by imaging applications, where such boundary curves represent edges of images. The shearlet approach is able to characterize both locations and orientations of the edge points, including corner points and junctions, where the edge curves exhibit abrupt changes in tangent or curvature. Our results encompass and greatly extend previous results based on the shearlet and curvelet transforms which were limited to very special cases such as polygons and smooth boundary curves with nonvanishing curvature.
\end{abstract}

Key words. Analysis of singularities, continuous wavelets, curvelets, directional wavelets, edge detection, shearlets, wavelets

AMS subject classifications. $42 \mathrm{C} 15,42 \mathrm{C} 40$

1. Introduction. Consider a function $f(x, y)$, on a bounded subset of $\mathbb{R}^{2}$, which is smooth apart from singularities along the curves $\gamma_{1}, \ldots, \gamma_{n}$. Our goal is to exactly identify the curves $\gamma_{1}, \ldots, \gamma_{n}$ and analyze their geometrical properties. This setting provides an idealized model for a large class of images $f$ where the singularity curves $\gamma_{1}, \ldots, \gamma_{n}$ represent the edges of the images. Since edges are the most prominent features in images, their detection and analysis is a major task in several applications from image processing and computer vision [3].

In this paper, we show that the shearlet transform, a novel multiscale directional transform introduced by the authors and their collaborators, provides a most powerful tool for the precise geometrical characterization of the edges of $f$. Our results encompass, extend and refine a number of observations appeared recently in the literature, which use the continuous wavelet transform and their generalizations to resolve the singularities of functions and distributions.

It is well-known that the continuous wavelet transform, which maps a function $f$ into a transform $\mathcal{W}_{\psi} f(a, t)$ depending on the scales $a>0$ and the locations $t$, has the ability to identify the set of singularities $f$. If $f$ is smooth apart from a discontinuity at a point $x_{0}$, then the continuous transform $\mathcal{W}_{\psi} f(a, t)$ decays rapidly as $a$ approaches 0 (fine scales), unless $t$ is near $x_{0}[10,13]$. Thus, the locations for the slow decay of $\mathcal{W}_{\psi} f(a, t)$, as $a \rightarrow 0$, can be used to resolve the singular support of $f$, that is, the set of points where $f$ is not regular. However, this standard multiscale approach is unable to provide additional information about the geometry of the set of singularities of $f$. In many situations, including the study of propagation of singularities in PDE problems or in imaging applications where one is concerned with the analysis and detection of edges, it is useful to not only identify their locations, but also their geometrical properties, such as tangent vectors and curvature.

Very recently, starting with the seminal work by Candès and Donoho on the curvelet transform [2] and later using our approach based on the shearlet transform [6,

\footnotetext{
*Department of Mathematics, Missouri State University, Springfield,Missouri 65804, USA ( KanghuiGuo@MissouriState.edu)

${ }^{\dagger}$ Department of Mathematics, North Carolina State University, Campus Box 8205, Raleigh, NC 27695, USA (dlabate@math.ncsu.edu)
} 
11], it was shown that, by appropriately combining the multiscale framework with the ability to capture anisotropic and directional features, it is possible to describe both the location and geometry of the set of singularities of functions and distributions. For example, the continuous curvelet and shearlet transforms are useful to characterize the wavefront set of a distribution $[2,11]$. Other results show that, for some special regions such as polygons and convex sets with boundary having non-vanishing curvature, the continuous shearlet transform precisely capture the geometric information of the boundary curves through its decay at fine scales [8]. Specifically, the transform decays rapidly at fine scales everywhere, except on the edge points, for normal orientation, in which case the decay is 'slow'. Notice that it was previoulsy observed in [1] that the curvelet transform of a function $f$ decays rapidly for locations $t$ where $f$ is regular; for $t$ on a singularity and normal orientation $\theta$, the (discrete) curvelet transform satisfies $\left|\left\langle f, \psi_{j, \theta, t}\right\rangle\right| \leq C 2^{-\frac{3}{4} j}$, at fine scales (see also [7]). This upper bound estimate, however, does not guarantee that the decay is necessarily as slow as $2^{-\frac{3}{4} j}$ at $t$. To effectively "detect" the discontinuity, an appropriate lower bound estimate is needed. This issue was addressed in [8].

In this paper, we extend the observations above to a much more general and "realistic" setting, by showing that the continuous shearlet transform is useful to characterize the boundary curves of very general planar regions. This includes not only smooth edge points, but also edge points with abrupt changes in the tangent or curvature. Indeed such points (frequently indicated as corner points or junctions) usually provide the most conspicuous and useful features for many algorithms of edge analysis and feature extraction $[17,12]$.

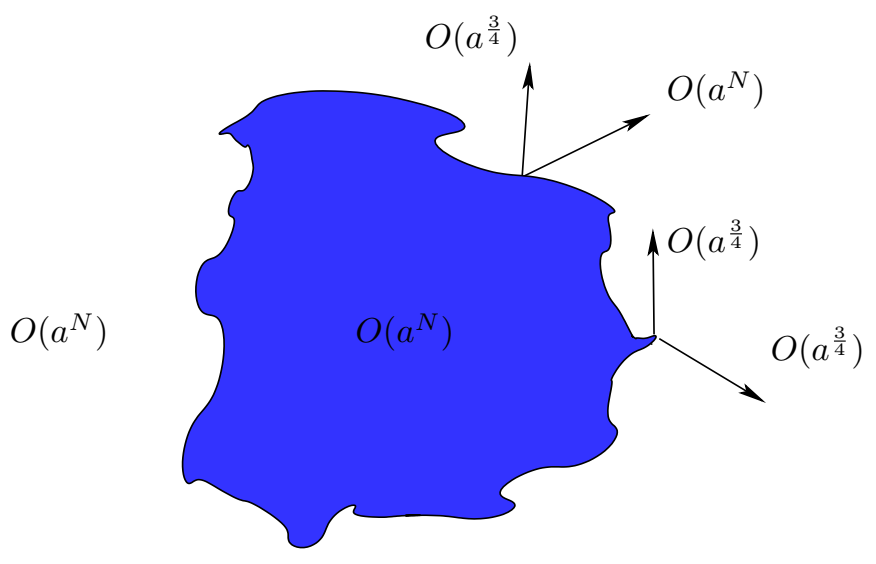

FIG. 1.1. Asymptotic decay of the continuous shearlet transform of the $B(x)=\chi_{S}(x)$. At the regular points $p \in \partial S$, for normal orientation, the shearlet transform decays as $O\left(a^{\frac{3}{4}}\right)$. For all other values of $s$, the decay is as fast as $O\left(a^{N}\right)$, for any $N \in \mathbb{N}$. At the corner points, the shearlet transform decays as $O\left(a^{\frac{3}{4}}\right)$ for both normal orientations.

To illustrate our results, let $S \subset \mathbb{R}^{2}$ and suppose that its boundary $\partial S$ is a piecewise smooth curve. Let $B=\chi_{S}$, and consider the continuous shearlet transform of $B$ :

$$
\mathcal{S H}_{\psi} B(a, s, p)=\left\langle B, \psi_{\text {asp }}\right\rangle, \quad a>0, s \in \mathbb{R}, p \in \mathbb{R}^{2} .
$$

This transform is mapping $B \in L^{2}\left(\mathbb{R}^{2}\right)$ into a collection of coefficients $\left\langle B, \psi_{\text {asp }}\right\rangle$ associated with scales $a>0$, orientations $s \in \mathbb{R}$ and locations $p \in \mathbb{R}^{2}$. This approach 
is a truly multidimensional extension of the classical continuous wavelet transform, providing a unique ability to capture the geometric information of multidimensional functions. Specifically, we will show that the asymptotic decay of $\mathcal{S H}_{\psi} B(a, s, p)$, at fine scale $a \rightarrow 0$, signals both the location and the orientation of the boundary curve $\partial S$. The decay properties of the continuous shearlet transform are illustrated in Figure 1.1, which shows that:

- If $p \notin \partial S$, then $\left|\mathcal{S H}_{\psi} B(a, s, p)\right|$ decays rapidly, as $a \rightarrow 0$, for each $s \in \mathbb{R}$.

- If $p \in \partial S$ and $\partial S$ is smooth near $p$, then $\left|\mathcal{S H}_{\psi} B(a, s, p)\right|$ decays rapidly, as $a \rightarrow 0$, for each $s \in \mathbb{R}$ unless $s=s_{0}$ is the normal orientation to $\partial S$ at $p$. In this last case, $\left|\mathcal{S H}_{\psi} B\left(a, s_{0}, p\right)\right| \sim a^{\frac{3}{4}}$, as $a \rightarrow 0$.

- If $p$ is a corner point of $\partial S$ and $s=s_{0}, s=s_{1}$ are the normal orientations to $\partial S$ at $p$, then $\left|\mathcal{S H}_{\psi} B\left(a, s_{0}, p\right)\right|,\left|\mathcal{S H}_{\psi} B\left(a, s_{1}, p\right)\right| \sim a^{\frac{3}{4}}$, as $a \rightarrow 0$. For all other orientations, the asymptotic decay of $\left|\mathcal{S H}_{\psi} B(a, s, p)\right|$ is faster (even if not necessarily "rapid").

Notice that, by "rapid decay", we mean that, given any $N \in \mathbb{N}$, there is a $C_{N}>0$ such that $\left|\mathcal{S H}_{\psi} B(a, s, p)\right| \leq C a^{N}$, as $a \rightarrow 0$. The more precise statements of these results, including the discussion of the corner points, will be given in Section 3.

Supported by the theoretical results presented in this paper, numerical algorithms for edge detection and identification of corner points have been developed by the authors and other collaborators and presented in a separate paper. The numerical tests clearly indicate that the shearlet approach outperforms wavelet-based and other traditional methods which do not have the same ability to extract the directional information of edges. We refer to [16] for extensive numerical demonstrations of the application of the shearlet transform to problems of edge analysis and detection.

The paper is organized as follows. In Section 2, we recall the definition of the continuous shearlet transform. Our main results are stated in Section 3. In Section 4 we develop some technical tools with are used in the proofs of our main theorems, presented in Section 5. Finally, in Section 6, we briefly describe a shearlet-based approach for the classification of some geometrical features in planar shapes.

2. The shearlet transform. We start by recalling the basic properties of the continuous shearlet transform, which was introduced in $[6,11]$. Consider the subspace of $L^{2}\left(\mathbb{R}^{2}\right)$ given by $L^{2}(C)^{\vee}=\left\{f \in L^{2}\left(\mathbb{R}^{2}\right)\right.$ : supp $\left.\hat{f} \subset C\right\}$, where $C$ is the "horizontal cone" in the frequency plane:

$$
C=\left\{\left(\xi_{1}, \xi_{2}\right) \in \mathbb{R}^{2}:\left|\xi_{1}\right| \geq 2 \text { and }\left|\frac{\xi_{2}}{\xi_{1}}\right| \leq 1\right\} .
$$

The following proposition, which is a simple generalization of a result from [11], provides sufficient conditions on the function $\psi$ for obtaining a reproducing system of continuous shearlets on $L^{2}(C)^{\vee}$.

Proposition 2.1. Consider the shearlet group $\Lambda^{(h)}=\left\{\left(M_{a s}, p\right): 0<a \leq\right.$ $\left.\frac{1}{4},-\frac{3}{2} \leq s \leq \frac{3}{2}, p \in \mathbb{R}^{2}\right\}$, where $M_{a s}=\left(\begin{array}{cc}a & -a^{1 / 2} s \\ 0 & a^{1 / 2}\end{array}\right)$. For $\xi=\left(\xi_{1}, \xi_{2}\right) \in \mathbb{R}^{2}, \xi_{2} \neq 0$, let $\psi^{(h)}$ be defined by

$$
\hat{\psi}^{(h)}(\xi)=\hat{\psi}^{(h)}\left(\xi_{1}, \xi_{2}\right)=\hat{\psi}_{1}\left(\xi_{1}\right) \hat{\psi}_{2}\left(\frac{\xi_{2}}{\xi_{1}}\right)
$$

where:

(i) $\psi_{1} \in L^{2}(\mathbb{R})$ satisfies the (generalized) Calderòn condition

$$
\int_{0}^{\infty}\left|\hat{\psi}_{1}(a \xi)\right|^{2} \frac{d a}{a}=1 \quad \text { for a.e. } \xi \in \mathbb{R}
$$


and supp $\hat{\psi}_{1} \subset\left[-2,-\frac{1}{2}\right] \cup\left[\frac{1}{2}, 2\right]$;

(ii) $\left\|\psi_{2}\right\|_{L^{2}}=1$ and supp $\hat{\psi}_{2} \subset[-1,1]$.

Then, for all $f \in L^{2}(C)^{\vee}$, we have

$$
f(x)=\int_{\mathbb{R}^{2}} \int_{-\frac{3}{2}}^{\frac{3}{2}} \int_{0}^{\frac{1}{4}}\left\langle f, \psi_{a s p}^{(h)}\right\rangle \psi_{a s p}^{(h)}(x) \frac{d a}{a^{3}} d s d p,
$$

where $\psi_{a s p}^{(h)}(x)=\left|\operatorname{det} M_{a s}\right|^{-\frac{1}{2}} \psi^{(h)}\left(M_{a s}^{-1}(x-p)\right)$, with convergence in the $L^{2}$ sense.

If the assumptions of Proposition 2.1 are satisfied, we say that the functions

$$
\Psi^{(h)}=\left\{\psi_{a s p}^{(h)}: 0<a \leq \frac{1}{4},-\frac{3}{2} \leq s \leq \frac{3}{2}, p \in \mathbb{R}^{2}\right\}
$$

are continuous shearlets for $L^{2}(C)^{\vee}$ and that the corresponding mapping from $f \in$ $L^{2}(C)^{\vee}$ into $\mathcal{S H}{ }^{(h)} f(a, s, p)=\left\langle f, \psi_{a s p}^{(h)}\right\rangle$ is the continuous shearlet transform on $L^{2}(C)^{\vee}$ with respect to $\Lambda^{(h)}$. The index label $(h)$ used in the notation of the shearlet system $\Psi^{(h)}$ (and the corresponding shearlet transform) indicates that the system (2.1) has frequency support in the horizontal cone $C$; a second shearlet system will be defined below with support in the complementary vertical cone.

Observe that, in the frequency domain, a shearlet $\psi_{a s p}^{(h)} \in \Psi^{(h)}$ has the form:

$$
\hat{\psi}_{a s p}^{(h)}\left(\xi_{1}, \xi_{2}\right)=a^{\frac{3}{4}} \hat{\psi}_{1}\left(a \xi_{1}\right) \hat{\psi}_{2}\left(a^{-\frac{1}{2}}\left(\frac{\xi_{2}}{\xi_{1}}-s\right)\right) e^{-2 \pi i \xi p} .
$$

Thus, each function $\hat{\psi}_{a s p}^{(h)}$ has support:

$$
\operatorname{supp} \hat{\psi}_{a s p}^{(h)} \subset\left\{\left(\xi_{1}, \xi_{2}\right): \xi_{1} \in\left[-\frac{2}{a},-\frac{1}{2 a}\right] \cup\left[\frac{1}{2 a}, \frac{2}{a}\right],\left|\frac{\xi_{2}}{\xi_{1}}-s\right| \leq a^{\frac{1}{2}}\right\} .
$$

That is, its frequency support is a pair of trapezoids, symmetric with respect to the origin, oriented along a line of slope $s$. The support becomes increasingly elongated as $a \rightarrow 0$. This is illustrated in Figure 1 .

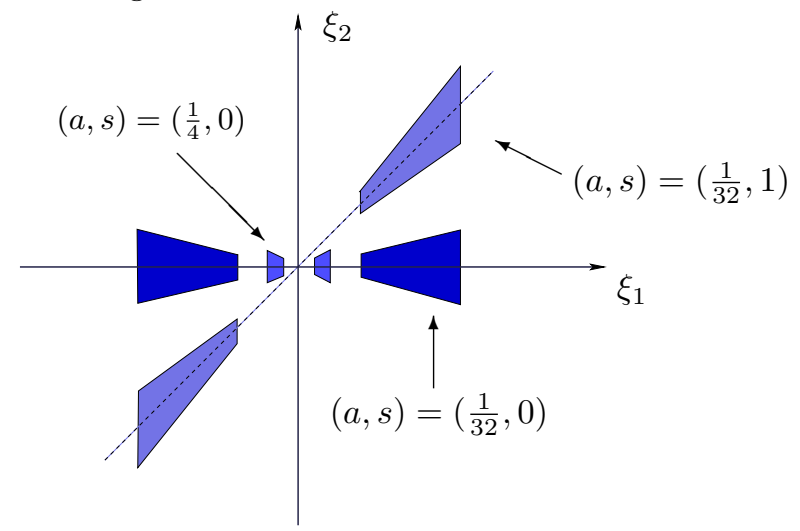

Fig. 2.1. Support of the shearlets $\hat{\psi}_{\text {asp }}$ (in the frequency domain) for different values of a and s.

There are a variety of examples of functions $\psi_{1}$ and $\psi_{2}$ satisfying the assumptions of Proposition 2.1. In particular, one can find a number of such examples with the additional property that $\hat{\psi}_{1}, \hat{\psi}_{2} \in C_{0}^{\infty}[6,11]$. For the kind of applications which will be described in this paper, some further additional properties are needed. In particular, we will require that $\hat{\psi}_{1}$ is a smooth odd function, and that $\hat{\psi}_{2}$ is an even smooth 
function which is decreasing on $[0,1)$. We refer to [8] for details about their constructions. In the following, throughout the paper, we will assume that the functions $\psi_{1}$ and $\psi_{2}$ satisfy these assumptions.

As shown by Proposition 2.1, the continuous shearlet transform $\mathcal{S H}_{\psi}^{(h)}$ provides a reproducing formula only for functions in a proper subspace of $L^{2}\left(\mathbb{R}^{2}\right)$. To extend the transform to all $f \in L^{2}\left(\mathbb{R}^{2}\right)$, we can introduce a similar transform to deal with the functions supported on the "vertical cone":

$$
C^{(v)}=\left\{\left(\xi_{1}, \xi_{2}\right) \in \mathbb{R}^{2}:\left|\xi_{2}\right| \geq 2 \text { and }\left|\frac{\xi_{2}}{\xi_{1}}\right|>1\right\} .
$$

Specifically, let

$$
\hat{\psi}^{(v)}(\xi)=\hat{\psi}^{(v)}\left(\xi_{1}, \xi_{2}\right)=\hat{\psi}_{1}\left(\xi_{2}\right) \hat{\psi}_{2}\left(\frac{\xi_{1}}{\xi_{2}}\right),
$$

where $\hat{\psi}_{1}, \hat{\psi}_{2}$ satisfy the same assumptions as in Proposition 2.1, and consider the shearlet group $\Lambda^{(v)}=\left\{\left(N_{a s}, p\right): 0<a \leq 1,-\frac{3}{2} \leq s \leq \frac{3}{2}, p \in \mathbb{R}^{2}\right\}$, where $N_{a s}=$ $\left(\begin{array}{cc}a^{1 / 2} & 0 \\ -a^{1 / 2} & s\end{array}\right)$. Then it is easy to verify that the functions

$$
\Psi^{(v)}=\left\{\psi_{a s p}^{(v)}=\left|\operatorname{det} N_{a s}\right|^{-\frac{1}{2}} \psi^{(v)}\left(N_{a s}^{-1}(x-p)\right): 0<a \leq \frac{1}{4},-\frac{3}{2} \leq s \leq \frac{3}{2}, p \in \mathbb{R}^{2}\right\}
$$

are continuous shearlets for $L^{2}\left(C^{(v)}\right)^{\vee}$. The corresponding transform $\mathcal{S H}_{\psi}^{(v)} f(a, s, p)=$ $\left\langle f, \psi_{a s p}^{(v)}\right\rangle$ is the continuous shearlet transform on $L^{2}(C)^{\vee}$ with respect to $\Lambda^{(v)}$. Finally, by introducing an appropriate window function $W$, we can represent the functions with frequency support on the set $[-2,2]^{2}$ as

$$
f=\int_{\mathbb{R}^{2}}\left\langle f, W_{p}\right\rangle W_{p} d p
$$

where $W_{p}(x)=W(x-p)$. As a result, we can represent any function $f \in L^{2}\left(\mathbb{R}^{2}\right)$ with respect of the full system of shearlets, which includes the set of horizontal shearlets $\psi_{a s p}^{(h)}$, vertical shearlets $\psi_{a s p}^{(v)}$ and coarse-scale isotropic functions $W_{p}$. We refer to [11] for more details about this representation. For our purposes, it is only the behavior of the fine-scale shearlets that matters. In this paper, we will apply the continuous shearlet transforms $\mathcal{S H}_{\psi}^{(h)}$ and $\mathcal{S H}_{\psi}^{(v)}$, at fine scales $(a \rightarrow 0)$, to resolve and precisely describe the boundaries of certain planar regions. Hence, it will be convenient to re-define the "fine-scale" shearlet transform as follows. For $0<a \leq 1 / 4, s \in \mathbb{R}$, $t \in \mathbb{R}^{2}$, the (fine-scale) continuous shearlet transform is the mapping from $f \in L^{2}\left(\mathbb{R}^{2} \backslash\right.$ $\left.[-2,2]^{2}\right)^{\vee}$ into $\mathcal{S H}_{\psi} f$ which is defined by:

$$
\mathcal{S H}_{\psi} f(a, s, p)= \begin{cases}\mathcal{S H}_{\psi}^{(h)}(a, s, p) & \text { if }|s| \leq 1 \\ \mathcal{S H}_{\psi}^{(v)}\left(a, \frac{1}{s}, p\right) & \text { if }|s|>1 .\end{cases}
$$

Remark. One can define a more general family of shearlet groups, associated with a parameter $0<\alpha<1$. They are defined by $\Lambda_{\alpha}=\left\{\left(M_{a s}, t\right): 0<a \leq \frac{1}{4},-\frac{3}{2} \leq\right.$ $\left.s \leq \frac{3}{2}, t \in \mathbb{R}^{2}\right\}$, where $M_{a s}=\left(\begin{array}{cc}a-a^{\alpha} s \\ 0 & a^{\alpha}\end{array}\right)$. Corresponding to these groups, one can define a family of Continuous $\alpha$-Shearlet Transforms $\mathcal{S H}_{\psi}^{(\alpha)} f$ with properties similar to those of the one we defined above (case $\alpha=1 / 2$ ). For simplicity of notation, in the following we will only consider the case $\alpha=1 / 2$. Our results presented below can be easily extended to the other values of $\alpha \in(0,1)$ (see [8], where the transform $\mathcal{S H}_{\psi}^{(\alpha)} f$ is applied to characterize the boundaries of convex boundaries with non-vanishing curvature). 
3. Main results. The main goal of this paper is to characterize the boundaries of bounded planar regions $S \subset \mathbb{R}^{2}$ by applying the continuous shearlet transform.

We assume that the boundary set of $S$, denoted by $\partial S$, is a simple curve, of finite length $L$, and is smooth except for finitely many 'corner points'. To precisely define such corner points, it is useful to introduce a parametrization of $\partial S$. Hence, let $\vec{\alpha}(t)$ be the parametrization of $\partial S$ with respect to the arc length parameter $t$. For any $t_{0} \in$ $(0, L)$ and any $j \geq 0$, we assume that $\lim _{t \rightarrow t_{0}^{-}} \vec{\alpha}^{(j)}(t)=\vec{\alpha}^{(j)}\left(t_{0}^{-}\right)$and $\lim _{t \rightarrow t_{0}^{+}} \vec{\alpha}^{(j)}(t)=$ $\vec{\alpha}^{(j)}\left(t_{0}^{+}\right)$exist. Also, let $\vec{n}\left(t^{-}\right), \vec{n}\left(t^{+}\right)$be the outer normal direction(s) of $\partial S$ at $\vec{\alpha}(t)$ from the left and right, respectively; if these two are equal, we will write them as $\vec{n}(t)$. Similarly, for the curvature of $\partial S$, we use notations $\kappa\left(t^{-}\right), \kappa\left(t^{+}\right)$and $\kappa(t)$.

We say that $p=\vec{\alpha}\left(t_{0}\right)$ is a corner point of $\partial S$ if either $(i) \quad \vec{\alpha}^{\prime}\left(t_{0}^{-}\right) \neq \pm \vec{\alpha}^{\prime}\left(t_{0}^{+}\right)$or (ii) $\vec{\alpha}^{\prime}\left(t_{0}^{-}\right)= \pm \vec{\alpha}^{\prime}\left(t_{0}^{+}\right)$, but $\kappa\left(t_{0}^{-}\right) \neq \kappa\left(t_{0}^{+}\right)$. When (i) holds, we say that $p$ is a corner point of first type, while when (ii) holds, we say that $p$ is a corner point of second type. On the other hand, if $\vec{\alpha}(t)$ is infinitely many times differentiable at $t_{0}$, we say that $\vec{\alpha}\left(t_{0}\right)$ is a regular point of $\partial S$. Finally, we say that the boundary curve $\vec{\alpha}(t)$ is piecewise smooth if the values $\vec{\alpha}(t)$ are regular points for all $0 \leq t \leq L$, except for finitely many corner points.

The above definition of piecewise smooth boundary curve can be modified by assuming that the regular points of $\partial S$ are $M$ times differentiable, for $M \in \mathbb{N}$, rather than infinitely differentiable. All the results presented below can be adapted to the case of piecewise $C^{M}$ boundary curves, for $M \geq 3$.

Let $p=\vec{\alpha}\left(t_{0}\right)$ be a regular point and let $s=\tan \left(\theta_{0}\right)$ with $\theta_{0} \in\left(-\frac{\pi}{2}, \frac{\pi}{2}\right)$. Let $\Theta\left(\theta_{0}\right)=\left(\cos \theta_{0}, \sin \theta_{0}\right)$. We say that $s$ corresponds to the normal direction of $\partial S$ at $p$ if $\Theta\left(\theta_{0}\right)= \pm \vec{n}\left(t_{0}\right)$. We can proceed similarly when $\vec{\alpha}\left(t_{0}\right)$ is a corner point. In this case, however, there are two outer normal directions $\vec{n}\left(t_{0}^{-}\right)$and $\vec{n}\left(t_{0}^{+}\right)$.

We are now ready to state our results.

If $p \notin \partial S$, it was shown in [8] that the continuous shearlet transform $\mathcal{S H}_{\psi} B(a, s, p)$ decays rapidly, asymptotically for $a \rightarrow 0$. That is, for any $s \in \mathbb{R}$,

$$
\lim _{a \rightarrow 0^{+}} a^{-N} \mathcal{S H}_{\psi} B\left(a, s_{0}, p\right)=0, \quad \text { for all } N>0 .
$$

On the other hand, if $p \in \partial S$ the asymptotic decay of the continuous shearlet transform $\mathcal{S H}_{\psi} B(a, s, p)$, as $a \rightarrow 0$, we have the following facts.

TheOREM 3.1. Let $p \in \partial S$ be a regular point.

(i) If $s=s_{0}$ does not correspond to the normal direction of $\partial S$ at $p$ then

$$
\lim _{a \rightarrow 0^{+}} a^{-N} \mathcal{S H}_{\psi} B\left(a, s_{0}, p\right)=0, \quad \text { for all } N>0
$$

(ii) If $s=s_{0}$ corresponds to the normal direction of $\partial S$ at $p$ then

$$
0<\lim _{a \rightarrow 0^{+}} a^{-\frac{3}{4}}\left|\mathcal{S H}_{\psi} B\left(a, s_{0}, p\right)\right|<\infty .
$$

TheOREM 3.2. Let $p \in \partial S$ be a corner point.

(i) If $p$ is a corner point of the first type and $s=s_{0}$ does not correspond to any of the normal directions of $\partial S$ at $p$, then

$$
\lim _{a \rightarrow 0^{+}} a^{-\frac{9}{4}}\left|\mathcal{S H}_{\psi} B\left(a, s_{0}, p\right)\right|<\infty .
$$


(ii) If $p$ is a corner point of the second type and $s=s_{0}$ does not correspond to any of the normal directions of $\partial S$ at $p$, then

$$
0<\lim _{a \rightarrow 0^{+}} a^{-\frac{9}{4}}\left|\mathcal{S H}_{\psi} B\left(a, s_{0}, p\right)\right|<\infty .
$$

(iii) If $s=s_{0}$ corresponds to one of the normal directions of $\partial S$ at $p$ then

$$
0<\lim _{a \rightarrow 0^{+}} a^{-\frac{3}{4}}\left|\mathcal{S H}_{\psi} B\left(a, s_{0}, p\right)\right|<\infty .
$$

Theorem 3.1 shows that, for $p \in \partial S$, the continuous shearlet transform decays rapidly, asymptotically for $a \rightarrow 0$, unless $s=s_{0}$ corresponds to the normal direction of $\partial S$ at $p$, in which case

$$
\left|\mathcal{S H}_{\psi} B\left(a, s_{0}, p\right)\right| \sim O\left(a^{\frac{3}{4}}\right), \text { as } a \rightarrow 0 .
$$

This generalizes the results found in $[8,11]$, which were limited to the case where $B=\chi_{S}$ was a disc or a convex set with non-vanishing curvature. In the case of the disc, a similar result, with the same decay rate, holds for the continuous curvelet transform [2].

Theorem 3.2 shows that, at a corner points $p$, the asymptotic decay of the continuous shearlet transform depends both on the tangent and the curvature at $p$. If $s=s_{0}$ corresponds to one of the normal directions of $\partial S$ at $p$, then the continuous shearlet transform decays as

$$
\left|\mathcal{S H}_{\psi} B\left(a, s_{0}, p\right)\right| \sim O\left(a^{\frac{3}{4}}\right), \text { as } a \rightarrow 0 .
$$

This is the same decay rate as for regular points, when $s_{0}$ corresponds to the normal direction (but now there are two normal directions). If $p$ is a corner point of the second type and $s$ does not correspond to any of the normal directions, then

$$
\left|\mathcal{S H}_{\psi} B\left(a, s_{0}, p\right)\right| \sim O\left(a^{\frac{9}{4}}\right), \text { as } a \rightarrow 0,
$$

which is a faster decay rate than in the normal-orientation case. Finally, if $p$ is a corner point of the first type and $s_{0}$ does not correspond to any of the normal directions, then, by the theorem, we only know that the asymptotic decay of $\left|\mathcal{S H}_{\psi} B\left(a, s_{0}, p\right)\right|$ is not slower than $O\left(a^{\frac{9}{4}}\right)$; however the decay could be faster than $O\left(a^{\frac{9}{4}}\right)$. For example, the following result shows what happens under certain special assumptions on the curvature $\kappa(t)$ at $p$.

Proposition 3.3. Let $p=\vec{\alpha}\left(t_{0}\right) \in \partial S$ be a corner point of the first type and suppose that $s=s_{0}$ does not correspond to any of the normal direction of $\partial S$ at $p$.

(i) If $\kappa\left(t_{0}^{-}\right) \kappa\left(t_{0}^{+}\right)=0$ and $\kappa\left(t_{0}^{-}\right)+\kappa\left(t_{0}^{+}\right)>0$, then

$$
0<\lim _{a \rightarrow 0^{+}} a^{-\frac{9}{4}}\left|\mathcal{S H}_{\psi} B\left(a, s_{0}, p\right)\right|<\infty .
$$

(ii) Assume that $\vec{\alpha}^{(j)}\left(t_{0}\right)=\overrightarrow{0}$ for all $j \geq 2$, then

$$
\lim _{a \rightarrow 0^{+}} a^{-N} \mathcal{S H}_{\psi} B\left(a, s_{0}, p\right)=0, \quad \text { for all } N>0 .
$$

By Proposition 3.3, the asymptotic decay of $\mathcal{S H}_{\psi} B\left(a, s_{0}, p\right)$, as $a \rightarrow 0$, at a corner $p$ is very different when $S$ is a half disk or $S$ is a polygon. In the case when $p$ is 
a corner point of a half disk (which satisfies the assumptions of Proposition 3.3(i)), when $s_{0}$ does not correspond to the normal directions, we have that

$$
\left|\mathcal{S H}_{\psi} B\left(a, s_{0}, p\right)\right| \sim O\left(a^{\frac{9}{4}}\right), \text { as } a \rightarrow 0 .
$$

However, when $S$ is $p$ is corner point of a polygon (which satisfies the assumptions of Proposition 3.3(ii)), when $s_{0}$ does not correspond to the normal directions, for any $N \in \mathbb{N}$ there is a constant $C_{N}>0$ such that

$$
\left|\mathcal{S H}_{\psi} B\left(a, s_{0}, p\right)\right| \leq C_{N} a^{N}, \text { as } a \rightarrow 0
$$

We want to point out that, for the continuous curvelet transform, when $s_{0}$ does not correspond to the normal directions, the corner points of either the half disk or the polygon have the same decay rate $O\left(a^{\frac{5}{4}}\right)$ as $a \rightarrow 0$. The reason behind this difference is that the affine-like structure of the shearlet transform yields some useful cancelation properties (see the remark made in the proof of Theorem 3.2); these properties do not hold for the curvelet transform.

4. Localization lemmata and other useful results. We will now establish a number of results providing the essential analytical tools to prove the main theorems of this paper. Lemmata 4.1 and 4.2 , in particular, show that the continuous shearlet transform exhibits precisely those localization properties which are needed to analyze the geometry of edges.

Let $S$ be a bounded planar regions whose boundary is smooth except for finitely many corner points, according to the definition in Section 3 . Let $B=\chi_{S}$.

By the divergence theorem, we can write the Fourier transform of $B$ as

$$
\begin{aligned}
\hat{B}(\xi) & =\widehat{\chi}_{S}(\xi)=-\frac{1}{2 \pi i|\xi|} \int_{\partial S} e^{-2 \pi i \xi x} \Theta(\theta) \cdot \vec{n}(x) d \sigma(x) \\
& =-\frac{1}{2 \pi i \rho} \int_{0}^{L} e^{-2 \pi i \rho \Theta(\theta) \cdot \vec{\alpha}(t)} \Theta(\theta) \cdot \vec{n}(t) d t
\end{aligned}
$$

where $\xi=\rho \Theta(\theta), \Theta(\theta)=(\cos \theta, \sin \theta)$. (Notice that this is the same approach which is used, for example, in [9]).

Using the expression above, we have that

$$
\begin{aligned}
& \mathcal{S} \mathcal{H}_{\psi} B(a, s, p) \\
& =\left\langle B, \psi_{\text {asp }}\right\rangle \\
& =\int_{0}^{2 \pi} \int_{0}^{\infty} \hat{B}(\rho, \theta) \overline{\hat{\psi}_{\text {asp }}^{(d)}}(\rho, \theta) \rho d \rho d \theta \\
& =-\frac{1}{2 \pi i} \int_{0}^{2 \pi} \int_{0}^{\infty} \int_{0}^{L} \overline{\hat{\psi}_{a s p}^{(d)}}(\rho, \theta) e^{-2 \pi i \rho \Theta(\theta) \cdot \vec{\alpha}(t)} \Theta(\theta) \cdot \vec{n}(t) d t d \rho d \theta,
\end{aligned}
$$

where the upper-script in $\psi_{a s p}^{(d)}$ is either $d=h$, when $|s| \leq 1$, or $d=v$, when $|s|>1$.

For $\epsilon>0$, let $D(\epsilon, p)$ be the ball in $\mathbb{R}^{2}$ of radius $\epsilon$ and center $p$, and $D^{c}(\epsilon, p)=$ $\mathbb{R}^{2} \backslash D(\epsilon, p)$. Hence, using (4.2), we can write the shearlet transform of $B$ as

$$
\mathcal{S H}_{\psi} B(a, s, p)=I_{1}(a, s, p)+I_{2}(a, s, p)
$$


where

$$
\begin{aligned}
& I_{1}(a, s, p) \\
& =-\frac{1}{2 \pi i} \int_{0}^{2 \pi} \int_{0}^{\infty} \int_{\partial S \cap D(\epsilon, p)} \overline{\hat{\psi}_{a s p}^{(d)}}(\rho, \theta) e^{-2 \pi i \rho \Theta(\theta) \cdot \vec{\alpha}(t)} \Theta(\theta) \cdot \vec{n}(t) d t d \rho d \theta \\
& I_{2}(a, s, p) \\
& =-\frac{1}{2 \pi i} \int_{0}^{2 \pi} \int_{0}^{\infty} \int_{\partial S \cap D^{c}(\epsilon, p)} \overline{\hat{\psi}_{a s p}^{(d)}}(\rho, \theta) e^{-2 \pi i \rho \Theta(\theta) \cdot \vec{\alpha}(t)} \Theta(\theta) \cdot \vec{n}(t) d t d \rho d \theta .
\end{aligned}
$$

The following lemma shows that the asymptotic decay of the shearlet transform $\mathcal{S H}_{\psi} B(a, s, p)$, as $a \rightarrow 0$, is only determined by the values of the boundary $\partial S$ which are "close" to $p$.

Lemma 4.1. Let $I_{2}(a, s, p)$ be given by (4.4). For any $N>$, there is a constant $C_{N}>0$ such that

$$
\left|I_{2}(a, s, p)\right| \leq C_{N} a^{N}
$$

asymptotically as $a \rightarrow 0$, uniformly for all $s \in \mathbb{R}$.

Proof. We will only examine the behavior of $I_{2}(a, s, p)$ for $|s| \leq 1$ (in which case we use the 'horizontal' shearlet transform). The case where $|s|>1$ is similar. We have:

$$
\begin{aligned}
I_{2}(a, s, p)= & -\frac{1}{2 \pi i} \int_{\partial S \cap D^{c}(\epsilon, p)} \int_{0}^{2 \pi} \int_{0}^{\infty} \overline{\hat{\psi}_{a s p}^{(h)}}(\rho, \theta) e^{-2 \pi i \rho \Theta(\theta) \cdot \vec{\alpha}(t)} \Theta(\theta) \cdot \vec{n}(t) d t d \rho d \theta \\
= & \frac{-a^{3 / 4}}{2 \pi i} \int_{\partial S \cap D^{c}(\epsilon, p)} \int_{0}^{2 \pi} \int_{0}^{\infty} \hat{\psi}_{1}(a \rho \cos \theta) \hat{\psi}_{2}\left(a^{-1 / 2}(\tan \theta-s)\right) \\
& \times e^{2 \pi i \rho \Theta(\theta) p} d \rho d \theta e^{-2 \pi i \rho \Theta(\theta) \cdot \vec{\alpha}(t)} \Theta(\theta) \cdot \vec{n}(t) d t \\
= & \frac{-a^{-1 / 4}}{2 \pi i} \int_{\partial S \cap D^{c}(\epsilon, p)} \int_{0}^{2 \pi} \int_{0}^{\infty} \hat{\psi}_{1}(\rho \cos \theta) \hat{\psi}_{2}\left(a^{-1 / 2}(\tan \theta-s)\right) \\
& \times e^{2 \pi i \frac{\rho}{a} \Theta(\theta) \cdot(p-\vec{\alpha}(t))} \Theta(\theta) \cdot \vec{n}(t) d \rho d \theta d t .
\end{aligned}
$$

By assumption, $\|p-\vec{\alpha}(t)\| \geq \epsilon$ for all $\vec{\alpha}(t) \in \partial S \cap D^{c}(\epsilon, p)$. Hence, there is a constant $C_{p}$ such that $\inf _{x \in \partial S \cap D^{c}(\epsilon, p)}|p-x|=C_{p}$. Let $\mathcal{I}=\left\{\theta:|\tan \theta-s| \leq a^{\frac{1}{2}}\right\}$, $\mathcal{I}_{1}=\left\{\theta:|\Theta(\theta) \cdot(p-x)| \geq \frac{C_{p}}{\sqrt{2}}\right\} \bigcap \mathcal{I}$, and $\mathcal{I}_{2}=\mathcal{I} \backslash \mathcal{I}_{1}$. Since the vectors $\Theta(\theta), \Theta^{\prime}(\theta)$ form an orthonormal basis in $\mathbb{R}^{2}$, it follows that, on the set $\mathcal{I}_{2}$, we have $\left|\Theta^{\prime}(\theta) \cdot(p-x)\right| \geq \frac{C_{p}}{\sqrt{2}}$. Hence we can express each one of the integrals $I_{21}$ as a sum of a term where $\theta \in \mathcal{I}_{1}$ and another term where $\theta \in \mathcal{I}_{2}$, and integrate by parts as follows. On $\mathcal{I}_{1}$, we integrate by parts with respect to the variable $\rho$; on $\mathcal{I}_{2}$ we integrate by parts with respect to the variable $\theta$. Doing this repeatedly, it yields that, for any positive integer $n$, $\left|I_{2}\right| \leq C_{n} a^{\frac{n}{2}}$. This finishes the proof of lemma 4.1.

When $p$ is a corner point of $\partial S$ and $s$ corresponds to one of the normal directions of $\partial S$ at $p$, it will be useful to replace a tiny portion of $\partial S$ around $p$ by appropriate line segments and arcs of circles. This is the motivation of Lemma 4.2. Before presenting this lemma, we need some notations.

Let $\vec{\alpha}(t)$ be the boundary curve $\partial S$, with $0 \leq t \leq L$. We may assume that $L>1$ and $p=(0,0)=\vec{\alpha}(1)$. We can write

$$
\mathcal{C}=\partial S \cap D(\epsilon,(0,0))=\mathcal{C}^{-} \cup \mathcal{C}^{+},
$$


where

$$
\mathcal{C}^{-}=\{\vec{\alpha}(t): 1-\epsilon \leq t \leq 1\}, \quad \mathcal{C}^{+}=\{\vec{\alpha}(t): 1 \leq t \leq 1+\epsilon\}
$$

According to the definition given above, if $\overrightarrow{\alpha^{(m)}}\left(1^{-}\right) \neq \pm \alpha^{(m)}\left(1^{+}\right)$for some $m \geq 1$, then $p=(0,0)$ is a $m$-corner point. Otherwise, if $\vec{\alpha}(t)$ is continuously differentiable at $t=1$, then $p$ is a regular point.

Let $\kappa\left(1^{-}\right), \kappa\left(1^{+}\right)$be the curvature of $\partial S$ at $p$ from the left and right respectively. If $\kappa\left(1^{-}\right)>0$, let $\widetilde{\mathcal{C}}^{-}=\{\vec{\beta}(t): 1-\epsilon \leq t \leq 1\}$ be an arc of the unique osculating circle of $\partial S$ at $p$ from the left. If, on the other hand, $\kappa\left(1^{-}\right)=0$, then let $\widetilde{\mathcal{C}}^{-}=\{\vec{\beta}(t)$ : $1-\epsilon \leq t \leq 1\}$ be a segment of the tangent line of $\partial S$ at $p$ from the left. In the proof of Lemma 4.2, we will use the fact that $\|\vec{\alpha}(t)-\vec{\beta}(t)\| \leq C(1-t)^{3}$ for all $t \in[1-\epsilon, 1]$. Also let $\widetilde{M}^{-}$to be the line segment connecting $\vec{\alpha}(1-\epsilon)$ to $\vec{\beta}(1-\epsilon)$. Similarly, we define the curve $\widetilde{\mathcal{C}}^{+}=\{\vec{\beta}(t): 1 \leq t \leq 1+\epsilon\}$ and the line segment $\widetilde{M}^{+}$which are associated with the curve $\mathcal{C}^{+}$. Finally, we set $\widetilde{\mathcal{C}}=\widetilde{\mathcal{C}}^{-} \cup \widetilde{\mathcal{C}}^{+}$and $\widetilde{M}=\widetilde{M}^{-} \cup \widetilde{M}^{+}$and we denote by $\widetilde{S}$ the planar region whose boundary is $\widetilde{S}=(\partial S \backslash \mathcal{C}) \cup(\widetilde{\mathcal{C}} \cup \widetilde{M})$, and $\widetilde{B}=\chi_{\widetilde{S}}$. That is, in a neighborhood of $(0,0)$, we have replaced the boundary $\partial S$, with unions of arcs and/or segments, depending on the values of the curvature of $\partial S$.

The following lemma adapts some ideas from the proof of Theorem $4.1 \mathrm{in}$ [8].

Lemma 4.2. Let $\widetilde{B}=\chi_{\widetilde{S}}$, where $\widetilde{S}$ is defined above. For any $s \in \mathbb{R}$, we have

$$
\lim _{a \rightarrow 0^{+}} a^{-\frac{3}{4}}\left|\mathcal{S H}_{\psi} B(a, s,(0,0))-\mathcal{S H}_{\psi} \widetilde{B}(a, s,(0,0))\right|=0 .
$$

Proof. Without loss of generality, we may assume $s=0$. Let $\gamma$ be chosen such that $\frac{3}{8}<\gamma<\frac{1}{2}$. Also one can assume that $a^{\gamma}<\epsilon$, so that $D\left(a^{\gamma},(0,0)\right) \subset D(\epsilon,(0,0))$ (and, hence, Lemma 4.1 applies).

A direct calculation shows that

$$
\begin{aligned}
\left|\mathcal{S H}_{\psi} B(a, 0,(0,0))-\mathcal{S H}_{\psi} \widetilde{B}(a, 0,(0,0))\right| & \leq \int_{\mathbb{R}^{2}}\left|\psi_{a 00}(x)\right|\left|\chi_{S}(x)-\chi_{\widetilde{S}}(x)\right| d x \\
& =T_{1}(a)+T_{2}(a),
\end{aligned}
$$

where, for $x=\left(x_{1}, x_{2}\right)$, we have:

$$
\begin{aligned}
& T_{1}(a)=a^{-\frac{3}{4}} \int_{D\left(a^{\gamma},(0,0)\right)}\left|\psi\left(a^{-1} x_{1}, a^{-\frac{1}{2}} x_{2}\right)\right|\left|\chi_{S}\left(x_{1}, x_{2}\right)-\chi_{\widetilde{S}}\left(x_{1}, x_{2}\right)\right| d x_{1} d x_{2} \\
& T_{2}(a)=a^{-\frac{3}{4}} \int_{D^{c}\left(a^{\gamma},(0,0)\right)}\left|\psi\left(a^{-1} x_{1}, a^{-\frac{1}{2}} x_{2}\right)\right|\left|\chi_{S}\left(x_{1}, x_{2}\right)-\chi_{\widetilde{S}}\left(x_{1}, x_{2}\right)\right| d x_{1} d x_{2} .
\end{aligned}
$$

Observe that:

$$
T_{1}(a) \leq C a^{-\frac{3}{4}} \int_{D\left(a^{\gamma},(0,0)\right)}\left|\chi_{S}\left(x_{1}, x_{2}\right)-\chi_{\widetilde{S}}\left(x_{1}, x_{2}\right)\right| d x_{1} d x_{2} .
$$

To estimate the above quantity, it is enough to compute the area between $S$ and $\widetilde{S}$. From the construction of $\widetilde{\mathcal{C}}$, we have

$$
\begin{aligned}
T_{1}(a) & \leq C a^{-\frac{3}{4}} \int_{1-a^{\gamma}}^{1+a^{\gamma}}\|\vec{\alpha}(t)-\vec{\beta}(t)\| d t \\
& \leq C a^{-\frac{3}{4}} \int_{1-a^{\gamma}}^{1+a^{\gamma}}|1-t|^{3} d t \\
& =C a^{4 \gamma-\frac{3}{4}} .
\end{aligned}
$$


Since $\gamma>\frac{3}{8}$, the above estimate shows that $T_{1}(a)=o\left(a^{\frac{3}{4}}\right)$.

The assumptions on $\psi$ imply that, for each $N>0$, there is a constant $C_{N}>0$ such that $|\psi(x)| \leq C_{N}\left(1+|x|^{2}\right)^{-N}$. Thus, for $a<1$, we can estimate $T_{2}(a)$ as:

$$
\begin{aligned}
T_{2}(a) & \leq C a^{-\frac{3}{4}} \int_{D^{c}\left(a^{\gamma},(0,0)\right)}\left|\psi\left(a^{-1} x_{1}, a^{-\frac{1}{2}} x_{2}\right)\right| d x_{1} d x_{2} \\
& \leq C_{N} a^{-\frac{3}{4}} \int_{D^{c}\left(a^{\gamma},(0,0)\right)}\left(1+\left(a^{-1} x_{1}\right)^{2}+\left(a^{-\frac{1}{2}} x_{2}\right)^{2}\right)^{-N} d x_{1} d x_{2} \\
& \leq C_{N} a^{-\frac{3}{4}} \int_{D^{c}\left(a^{\gamma},(0,0)\right)}\left(\left(a^{-1 / 2} x_{1}\right)^{2}+\left(a^{-\frac{1}{2}} x_{2}\right)^{2}\right)^{-N} d x_{1} d x_{2} \\
& =C_{N} a^{N-\frac{3}{4}} \int_{D^{c}\left(a^{\gamma},(0,0)\right)}\left(x_{1}^{2}+x_{2}^{2}\right)^{-N} d x_{1} d x_{2} \\
& =C_{N} a^{N-\frac{3}{4}} \int_{a^{\gamma}}^{\infty} r^{-1-2 N} d r \\
& =C_{N} a^{2 N\left(\frac{1}{2}-\gamma\right)+2 \gamma-\frac{3}{4}} .
\end{aligned}
$$

Since $\gamma<\frac{1}{2}$ and $N$ can be chosen arbitrarily large, then $T_{2}(a)=o\left(a^{\frac{3}{4}}\right)$.

The following result is a special case of the method of stationary phase (Proposition 8.3 from [14]).

Lemma 4.3. Let $\phi$ and $\psi$ be smooth functions. Suppose $\phi^{\prime}\left(x_{0}\right)=0$ and $\phi^{\prime \prime}\left(x_{0}\right) \neq$ 0. If $\psi$ is supported in a sufficiently small neighborhood of $x_{0}$, then

$$
J(\lambda)=\int_{\mathbb{R}^{n}} e^{i \lambda \phi(x)} \psi(x) d x=a_{0} e^{i \phi\left(x_{0}\right)} \lambda^{-1 / 2}+O\left(\lambda^{-1}\right),
$$

as $\lambda \rightarrow \infty$, where

$$
a_{0}=\left(\frac{2 \pi i}{\phi^{\prime \prime}\left(x_{0}\right)}\right)^{\frac{1}{2}} \psi\left(x_{0}\right)
$$

Finally, the following technical result follows by adapting an argument from [8]. For completeness, its proof is reported in Appendix A.

Lemma 4.4. Let $\psi_{2} \in L^{2}(\mathbb{R})$ be such that $\left\|\psi_{2}\right\|_{2}=1$, supp $\hat{\psi}_{2} \subset[-1,1]$ and $\hat{\psi}_{2}$ is even and decreasing on $[0,1]$. Then, for each $\rho>0$, one has

$$
\int_{0}^{1} \hat{\psi}_{2}(u)\left(\sin \left(\pi \rho u^{2}\right)+\cos \left(\pi \rho u^{2}\right)\right) d u>0
$$

Notice that the assumptions above on $\psi_{2}$ are the same as those we made in Section 2 .

5. Proofs of Main Theorems. We start by proving Theorem 3.2.

5.1. Proof of Theorem 3.2. (Part (i)). Assume that $s=s_{0}$ does not correspond to any of the normal directions of $\partial S$ at $p=(0,0)$. Let $s_{0}=\tan \theta_{0}$. In the following, we will assume that $\left|\theta_{0}\right| \leq \frac{\pi}{4}$. Otherwise, for the case $\frac{\pi}{4}<\left|\theta_{0}\right| \leq \frac{\pi}{2}$, we can proceed very similarly, after replacing the "horizontal" shearlet transform, with the "vertical" shearlet transform. Since the analysis of this situation is very similar, it will be omitted. 
Cutting $\left[-\frac{\pi}{2}, \frac{3 \pi}{2}\right]$ into $\left[-\frac{\pi}{2}, \frac{\pi}{2}\right]$ and $\left[\frac{\pi}{2}, \frac{3 \pi}{2}\right]$, and changing the variable $\theta=\theta^{\prime}+\pi$ for the integral on $\left[\frac{\pi}{2}, \frac{3 \pi}{2}\right]$, we can write $I_{1}$, given by (4.3), as

$$
I_{1}\left(a, s_{0},(0,0)\right)=I_{11}\left(a, s_{0},(0,0)\right)+I_{12}\left(a, s_{0},(0,0)\right),
$$

where, for $j=1,2$,

$$
\begin{aligned}
& I_{1 j}\left(a, s_{0},(0,0)\right) \\
& \quad=-\frac{a^{-\frac{1}{4}}}{2 \pi i} \int_{0}^{\infty} \int_{-\frac{\pi}{2}}^{\frac{\pi}{2}} \hat{\psi}_{1}(\rho \cos \theta) \hat{\psi}_{2}\left(a^{-1 / 2}\left(\tan \theta-\tan \theta_{0}\right)\right) K_{j}(a, \rho, \theta) d \theta d \rho,
\end{aligned}
$$

and

$$
K_{j}(a, \rho, \theta)=K_{j 1}(a, \rho, \theta)+K_{j 2}(a, \rho, \theta)
$$

with

$$
\begin{aligned}
& K_{11}(a, \rho, \theta)=\int_{1-\epsilon}^{1} e^{-2 \pi i \frac{\rho}{a} \Theta(\theta) \cdot \vec{\alpha}(t)} \Theta(\theta) \cdot n(t) d t, \\
& K_{12}(a, \rho, \theta)=\int_{1}^{1+\epsilon} e^{-2 \pi i \frac{\rho}{a} \Theta(\theta) \cdot \vec{\alpha}(t)} \Theta(\theta) \cdot n(t) d t, \\
& K_{21}(a, \rho, \theta)=\int_{1-\epsilon}^{1} e^{2 \pi i \frac{\rho}{a} \Theta(\theta) \cdot \vec{\alpha}(t)} \Theta(\theta) \cdot n(t) d t, \\
& K_{22}(a, \rho, \theta)=\int_{1}^{1+\epsilon} e^{2 \pi i \frac{\rho}{a} \Theta(\theta) \cdot \vec{\alpha}(t)} \Theta(\theta) \cdot n(t) d t .
\end{aligned}
$$

By the support condition on $\hat{\psi}_{2}$, we have that $\theta \rightarrow \theta_{0}$ as $a \rightarrow 0$. Since $s_{0}=\tan \theta_{0}$ does not correspond to any of the normal directions of $\partial S$ at $(0,0)$, it follows that $\Theta\left(\theta_{0}\right) \cdot \vec{\alpha}^{\prime}(1) \neq 0$. Hence, for $a$ sufficiently small (in which case $\theta$ is near $\theta_{0}$ ), there is an $\epsilon>0$ sufficiently small, such that $\Theta(\theta) \cdot \vec{\alpha}^{\prime}(t) \neq 0$ for all $\theta$ neat $\theta_{0}$ and $t \in[1-\epsilon, 1+\epsilon]$.

Writing

$$
e^{-2 \pi i \frac{\rho}{a} \Theta(\theta) \cdot \vec{\alpha}(t)}=\frac{-a}{2 \pi i \rho \Theta(\theta) \cdot \vec{\alpha}^{\prime}(t)}\left(e^{-2 \pi i \frac{\rho}{a} \Theta(\theta) \cdot \vec{\alpha}(t)}\right)^{\prime}
$$

and then integrating by parts twice the integral $K_{11}$, with respect to $t$, we obtain

$$
\begin{aligned}
K_{11}(a, \rho, \theta) & =-\frac{a}{2 \pi i \rho} \int_{1-\epsilon}^{1}\left(e^{-2 \pi i \frac{\rho}{a} \Theta(\theta) \cdot \vec{\alpha}(t)}\right)^{\prime} \frac{\Theta(\theta) \cdot \vec{n}(t)}{\Theta(\theta) \cdot \vec{\alpha}^{\prime}(t)} d t \\
& =K_{111}(a, \rho, \theta)+K_{112}(a, \rho, \theta)+K_{113}(a, \rho, \theta)+O\left(a^{3}\right),
\end{aligned}
$$

where

$$
\begin{aligned}
& K_{111}(a, \rho, \theta)=-\frac{a}{2 \pi i \rho} e^{-2 \pi i \frac{\rho}{a} \Theta(\theta) \cdot \vec{\alpha}\left(1^{-}\right)} \frac{\Theta(\theta) \cdot \vec{n}\left(1^{-}\right)}{\Theta(\theta) \cdot \vec{\alpha}^{\prime}\left(1^{-}\right)} \\
& K_{112}(a, \rho, \theta)=\frac{a}{2 \pi i \rho} e^{-2 \pi i \frac{\rho}{a} \Theta(\theta) \cdot \vec{\alpha}(1-\epsilon)} \frac{\Theta(\theta) \cdot \vec{n}(1-\epsilon)}{\Theta(\theta) \cdot \vec{\alpha}^{\prime}(1-\epsilon)} \\
& K_{113}(a, \rho, \theta)=\left.\frac{a^{2}}{(2 \pi i \rho)^{2}}\left(e^{-2 \pi i \frac{\rho}{a} \Theta(\theta) \cdot \vec{\alpha}(t)} \frac{1}{\Theta(\theta) \cdot \vec{\alpha}^{\prime}(t)}\left(\frac{\Theta(\theta) \cdot \vec{n}(t)}{\Theta(\theta) \cdot \vec{\alpha}^{\prime}(t)}\right)^{\prime}\right)\right|_{1-\epsilon} ^{1} .
\end{aligned}
$$


Similarly, one can write

$$
K_{12}(a, \rho, \theta)=K_{121}(a, \rho, \theta)+K_{122}(a, \rho, \theta)+K_{123}(a, \rho, \theta)+O\left(a^{3}\right) .
$$

Accordingly, we can write

$$
I_{11}\left(a, s_{0}, p\right)=I_{111}\left(a, s_{0}, p\right)+I_{112}\left(a, s_{0}, p\right)+I_{113}\left(a, s_{0}, p\right)+O\left(a^{3}\right),
$$

where, for $l=1,2,3$,

$$
\begin{aligned}
I_{11 l}\left(a, s_{0}, p\right)= & -\frac{a^{-\frac{1}{4}}}{2 \pi i} \int_{0}^{\infty} \int_{0}^{2 \pi} \hat{\psi}_{1}(\rho \cos \theta) \hat{\psi}_{2}\left(a^{-1 / 2}\left(\tan \theta-\tan \theta_{0}\right)\right) \times \\
& \times\left(K_{11 l}(a, \rho, \theta)+K_{12 l}(a, \rho, \theta)\right) d \theta d \rho .
\end{aligned}
$$

Similarly for the integral $I_{12}$, we can write

$$
I_{12}\left(a, s_{0}, p\right)=I_{121}\left(a, s_{0}, p\right)+I_{122}\left(a, s_{0}, p\right)+I_{123}\left(a, s_{0}, p\right)+O\left(a^{3}\right),
$$

where, for $l=1,2,3$,

$$
\begin{aligned}
I_{12 l}\left(a, s_{0}, p\right)= & -\frac{a^{-\frac{1}{4}}}{2 \pi i} \int_{0}^{\infty} \int_{0}^{2 \pi} \hat{\psi}_{1}(\rho \cos \theta) \hat{\psi}_{2}\left(a^{-1 / 2}\left(\tan \theta-\tan \theta_{0}\right)\right) \times \\
& \times\left(K_{21 l}(a, \rho, \theta)+K_{22 l}(a, \rho, \theta)\right) d \theta d \rho,
\end{aligned}
$$

and the terms $K_{21 l}, K_{22 l}$ are constructed as the corresponding terms $K_{11 l}, K_{12 l}$. It is easy to see that

$$
K_{111}(a, \rho, \theta)+K_{121}(a, \rho, \theta)+K_{211}(a, \rho, \theta)+K_{221}(a, \rho, \theta)=0 ;
$$

hence it follows that ${ }^{1}$

$$
I_{111}\left(a, s_{0}, p\right)+I_{121}\left(a, s_{0}, p\right)=0 .
$$

Since $\partial S$ is simple, it follows that $\vec{\alpha}(1-\epsilon) \neq(0,0)$ and $\vec{\alpha}(1+\epsilon) \neq(0,0)$. Therefore, by the argument in Lemma 4.1, we have that, for any $N>0$,

$$
\left|I_{112}\left(a, s_{0}, p\right)\right| \leq C_{N} a^{N}, \quad \text { as } a \rightarrow 0 .
$$

similarly one has

$$
\left|I_{122}\left(a, s_{0}, p\right)\right| \leq C_{N} a^{N}, \quad \text { as } a \rightarrow 0 .
$$

It only remains to analyze the terms $I_{113}, I_{123}$. To do that, notice that each one of the elements $K_{113}, K_{123}, K_{213}, K_{223}$, is made out of two terms, one at $t=1 \pm \epsilon$ and one at $t=1$. Similarly to $I_{112}\left(a, s_{0}, p\right)$ and $I_{122}\left(a, s_{0}, p\right)$, the terms evaluated at $t=1 \pm \epsilon$ have fast asymptotic decay as $a \rightarrow 0$, and can be included in negligible part $O\left(a^{3}\right)$. Thus, in order to determine the asymptotic decay rate for $I_{113}\left(a, s_{0}, p\right)+I_{123}\left(a, s_{0}, p\right)$, one only needs to analyze the corresponding $K_{i j k}$ terms at $t=1$.

\footnotetext{
${ }^{1}$ Here the the assumption that $\hat{\psi}_{1}$ is odd makes this cancelation possible. Notice that the corresponding generating function in the curvelet system is only defined in the radial direction and, hence, does not have the same cancelation property.
} 
Let $\kappa(t)$ be the curvature of $\partial S$ at $\vec{\alpha}(t)$. According to the Frenet formulas [4], we have that $\vec{\alpha}^{\prime \prime}(t)=\kappa(t) \vec{n}(t)$ and $\vec{n}^{\prime}(t)=-\kappa(t) \vec{\alpha}^{\prime}(t)$. Hence, using these formulas and the fact that the pair $\left\{\vec{\alpha}^{\prime}(t), \vec{n}(t)\right\}$ is an orthonormal basis in $R^{2}$, we have:

$$
\begin{aligned}
& \left(\frac{\Theta(\theta) \cdot \vec{n}(t)}{\Theta(\theta) \cdot \vec{\alpha}^{\prime}(t)}\right)^{\prime} \\
& =\frac{\left(\Theta(\theta) \cdot \vec{n}^{\prime}(t)\right)\left(\Theta(\theta) \cdot \vec{\alpha}^{\prime}(t)\right)-\left(\Theta(\theta) \cdot \vec{\alpha}^{\prime \prime}(t)\right)(\Theta(\theta) \cdot \vec{n}(t))}{\left(\Theta(\theta) \cdot \vec{\alpha}^{\prime}(t)\right)^{2}} \\
& =-\frac{\kappa(t)\left(\left(\Theta(\theta) \cdot \vec{\alpha}^{\prime}(t)\right)\left(\Theta(\theta) \cdot \vec{\alpha}^{\prime}(t)\right)+(\Theta(\theta) \cdot \vec{n}(t))(\Theta(\theta) \cdot \vec{n}(t))\right)}{\left(\Theta(\theta) \cdot \vec{\alpha}^{\prime}(t)\right)^{2}} \\
& =-\frac{\kappa(t)|\Theta(\theta)|^{2}}{\left(\Theta(\theta) \cdot \vec{\alpha}^{\prime}(t)\right)^{2}}=-\frac{\kappa(t)}{\left(\Theta(\theta) \cdot \vec{\alpha}^{\prime}(t)\right)^{2}}
\end{aligned}
$$

It follows from the above observations that

$$
\begin{aligned}
& \lim _{a \rightarrow 0^{+}}\left(\frac{\rho}{a}\right)^{2}\left(K_{113}^{-}\left(a, s_{0}, p\right)+K_{123}^{+}\left(a, s_{0}, p\right)\right) \\
& =\frac{1}{(2 \pi i)^{2}}\left(\frac{\kappa\left(1^{+}\right)}{\left(\Theta\left(\theta_{0}\right) \cdot \vec{\alpha}^{\prime}\left(1^{+}\right)\right)^{3}}-\frac{\kappa\left(1^{-}\right)}{\left(\Theta\left(\theta_{0}\right) \cdot \vec{\alpha}^{\prime}\left(1^{-}\right)\right)^{3}}\right) .
\end{aligned}
$$

Similarly one has

$$
\begin{aligned}
& \lim _{a \rightarrow 0^{+}}\left(\frac{\rho}{a}\right)^{2}\left(K_{213}^{-}\left(a, s_{0}, p\right)+K_{223}^{+}\left(a, s_{0}, p\right)\right) \\
& =\frac{1}{(2 \pi i)^{2}}\left(\frac{\kappa\left(1^{+}\right)}{\left(\Theta\left(\theta_{0}\right) \cdot \vec{\alpha}^{\prime}\left(1^{+}\right)\right)^{3}}-\frac{\kappa\left(1^{-}\right)}{\left(\Theta\left(\theta_{0}\right) \cdot \vec{\alpha}^{\prime}\left(1^{-}\right)\right)^{3}}\right) .
\end{aligned}
$$

Now by making the change of variables $u=a^{-\frac{1}{2}}\left(\tan \theta-\tan \theta_{0}\right)$ in $I_{113}$ and $I_{123}$, and applying (5.1) and (5.2), we obtain

$$
\lim _{a \rightarrow 0^{+}} a^{-\left(\frac{1}{4}+2\right)}\left(I_{113}\left(a, s_{0}, p\right)+I_{123}\left(a, s_{0}, p\right)\right)=-A,
$$

where

$$
\begin{gathered}
A=\frac{\cos ^{2} \theta_{0}}{2 \pi^{2}}\left(\frac{\kappa\left(1^{+}\right)}{\left(\Theta\left(\theta_{0}\right) \cdot \vec{\alpha}^{\prime}\left(1^{+}\right)\right)^{3}}-\frac{\kappa\left(1^{-}\right)}{\left(\Theta\left(\theta_{0}\right) \cdot \vec{\alpha}^{\prime}\left(1^{-}\right)\right)^{3}}\right) \\
\quad \times \int_{0}^{\infty} \hat{\psi}_{1}\left(\rho \cos \theta_{0}\right) d \rho \int_{-1}^{1} \hat{\psi}_{2}(u) d u .
\end{gathered}
$$

This completes the proof of part (i) of Theorem 3.2.

(Part (ii)) It is enough for us to show that $A \neq 0$, where $A$ is given by (5.3).

In this case, we have that

$$
\vec{\alpha}^{\prime}\left(1^{+}\right)=\vec{\alpha}^{\prime}\left(1^{-}\right) \quad \text { or } \quad \vec{\alpha}^{\prime}\left(1^{+}\right)=-\vec{\alpha}^{\prime}\left(1^{-}\right) .
$$

If $\vec{\alpha}^{\prime}\left(1^{+}\right)=\vec{\alpha}^{\prime}\left(1^{-}\right)$, from $\kappa\left(1^{+}\right) \neq \kappa\left(1^{-}\right)$, we have

$$
A=\frac{\cos ^{2} \theta_{0}}{2 \pi^{2}} \frac{\kappa\left(1^{+}\right)-\kappa\left(1^{-}\right)}{\left(\Theta\left(\theta_{0}\right) \cdot \vec{\alpha}^{\prime}(1)\right)^{3}} \int_{0}^{\infty} \hat{\psi}_{1}\left(\rho \cos \theta_{0}\right) d \rho \int_{-1}^{1} \hat{\psi}_{2}(u) d u \neq 0 .
$$


If on the other hand, $\vec{\alpha}^{\prime}\left(1^{+}\right)=-\vec{\alpha}^{\prime}\left(1^{-}\right)$, then it follows that $\vec{n}\left(1^{+}\right)=-\vec{n}\left(1^{-}\right)$. Since $\vec{\alpha}^{\prime \prime}\left(1^{+}\right)=-\kappa\left(1^{+}\right) \vec{n}\left(1^{-}\right)$and $\vec{\alpha}^{\prime \prime}\left(1^{-}\right)=\kappa\left(1^{-}\right) \vec{n}\left(1^{-}\right)$, and since $\kappa\left(1^{+}\right) \neq \kappa\left(1^{-}\right)$, we see that it is not possible to have $\kappa\left(1^{+}\right)=\kappa\left(1^{-}\right)=0$. Since we know $\kappa\left(1^{+}\right) \geq 0$, and $\kappa\left(1^{-}\right) \geq 0$, it follows that $\kappa\left(1^{+}\right)+\kappa\left(1^{-}\right)>0$.

Thus we have

$$
A=\frac{\cos ^{2} \theta_{0}}{2 \pi^{2}}\left(\frac{\kappa\left(1^{+}\right)+\kappa\left(1^{-}\right)}{\left(\Theta\left(\theta_{0}\right) \cdot \vec{\alpha}^{\prime}\left(1^{+}\right)\right)^{3}} \int_{0}^{\infty} \hat{\psi}_{1}\left(\rho \cos \theta_{0}\right) d \rho \int_{-1}^{1} \hat{\psi}_{2}(u) d u \neq 0 .\right.
$$

This completes the proof for (ii) of the theorem.

(Part (iii)). By the assumptions on the corner points, we see that if $s$ corresponds to the normal orientation to $C^{-}$at $p$, then it cannot correspond to the normal orientation to $C^{+}$at $p$. By part (i), it is enough to consider $C^{-}$or $C^{+}$. Thus, we may assume that $s$ corresponds to the outer normal direction to $\mathcal{C}^{-}$at $p=\vec{\alpha}(1)=(0,0)$. Without loss of generality, one may assume that $\vec{\alpha}^{\prime}\left(1^{-}\right)=(1,0)$ so that $\left.\vec{n}^{(} 1^{-}\right)=(0,1)$ (by rotating the coordinates if necessary).

Case 1: $\kappa\left(1^{-}\right)>0$.

According to Lemma 4.2, we may replace $\mathcal{C}^{-}$with $\vec{\beta}(t)$, a tiny arc of the osculating circle of $\mathcal{C}^{-}$at $p=\vec{\alpha}(1)=(0,0)$. We observe that in this setting, $\vec{n}(t)=\Theta(\phi)$, $d t=\frac{1}{\kappa(1)} d \phi$ and $\vec{\beta}(\phi)=\left(0, \frac{1}{\kappa\left(1^{-}\right)}\right)+\frac{1}{\kappa\left(1^{-}\right)} \Theta(\phi)$ with $\phi \in[-\pi / 2-\delta,-\pi / 2]$, where $\delta=\arcsin (\epsilon \kappa(1))$. Omitting faster decaying terms, we can write:

$$
\begin{aligned}
K^{-}(a, \rho, \theta) & =\int_{-\pi / 2-\delta}^{-\pi / 2} e^{-2 \pi i \frac{\rho}{a} \Theta(\theta) \cdot\left(x_{0}+\frac{1}{\kappa(1)} \Theta(\phi)\right)} \Theta(\theta) \cdot \Theta(\phi) \frac{1}{\kappa(1)} d \phi, \\
& =\frac{1}{\kappa(1)} e^{-2 \pi i \frac{\rho}{a} \frac{1}{\kappa(1)} \sin \theta} \int_{-\pi / 2-\delta}^{-\pi / 2} e^{-2 \pi i \frac{\rho}{a} \frac{1}{\kappa(1)} \cos (\theta-\phi)} \cos (\theta-\phi) d \phi, \\
& =\frac{1}{\kappa(1)} e^{-2 \pi i \frac{\rho}{a} \frac{1}{\kappa(1)} \sin \theta} \int_{\pi / 2+\theta}^{\pi / 2+\theta+\delta} e^{-2 \pi i \frac{\rho}{a} \frac{1}{\kappa(1)} \cos (\phi)} \cos (\phi) d \phi .
\end{aligned}
$$

Let

$$
I_{1}^{-}(a, \infty, 0)=-\frac{a^{-\frac{1}{4}}}{2 \pi i} \int_{0}^{\infty} \int_{0}^{2 \pi} \hat{\psi}_{1}(\rho \sin \theta) \hat{\psi}_{2}\left(a^{-1 / 2} \cot \theta\right) K^{-}(a, \rho, \theta) d \theta d \rho .
$$

Thus, using the expression of $K^{-}(a, \rho, \theta)$, and the change of variable $\theta \rightarrow \theta+\pi / 2$, one obtains:

$$
\begin{aligned}
& I_{1}^{-}(a, \infty, 0)=-\frac{a^{-\frac{1}{4}}}{2 \pi i \kappa\left(1^{-}\right)} \int_{0}^{\infty} \int_{0}^{2 \pi} \hat{\psi}_{1}(\rho \sin \theta) \hat{\psi}_{2}\left(a^{-1 / 2} \cot \theta\right) e^{-2 \pi i \frac{\rho}{a} \frac{1}{\kappa\left(1^{-}\right)} \sin \theta} \\
& \times \int_{\pi / 2+\theta}^{\pi / 2+\theta+\delta} e^{-2 \pi i \frac{\rho}{a} \frac{1}{\kappa(1-)} \cos (\phi)} \cos (\phi) d \phi d \theta d \rho \\
& =-\frac{a^{-\frac{1}{4}}}{2 \pi i \kappa\left(1^{-}\right)} \int_{0}^{\infty} \int_{0}^{2 \pi} \hat{\psi}_{1}(-\rho \cos \theta) \hat{\psi}_{2}\left(-a^{-1 / 2} \tan \theta\right) e^{2 \pi i \frac{\rho}{a} \frac{1}{\kappa\left(1^{-}\right)} \cos \theta} \\
& \times \int_{\theta}^{\theta+\delta} e^{-2 \pi i \frac{\rho}{a} \frac{1}{\kappa(1-)} \cos (\phi)} \cos (\phi) d \phi d \theta d \rho \\
& =T_{1}(a, \infty, 0)+T_{2}(a, \infty, 0),
\end{aligned}
$$


where

$$
\begin{aligned}
& T_{1}(a, \infty, 0)=\frac{a^{-\frac{1}{4}}}{2 \pi i \kappa\left(1^{-}\right)} \int_{0}^{\infty} \int_{-\pi / 2}^{\pi / 2} \hat{\psi}_{1}(\rho \cos \theta) \hat{\psi}_{2}\left(a^{-1 / 2} \tan \theta\right) e^{2 \pi i \frac{\rho}{a} \frac{1}{\kappa\left(1^{-}\right)} \cos \theta} \\
& \times \int_{\theta}^{\theta+\delta} e^{\left.-2 \pi i \frac{\rho}{a} \frac{1}{\kappa\left(1^{-}\right)} \cos (\phi)\right)} \cos (\phi) d \phi d \theta d \rho \\
& T_{2}(a, \infty, 0)=\frac{a^{-\frac{1}{4}}}{2 \pi i \kappa\left(1^{-}\right)} \int_{0}^{\infty} \int_{\pi / 2}^{3 \pi / 2} \hat{\psi}_{1}(\rho \cos \theta) \hat{\psi}_{2}\left(a^{-1 / 2} \tan \theta\right) e^{2 \pi i \frac{\rho}{a} \frac{1}{\kappa\left(1^{-}\right)} \cos \theta} \\
& \times \int_{\theta}^{\theta+\delta} e^{\left.-2 \pi i \frac{\rho}{a} \frac{1}{\kappa\left(1^{-}\right)} \cos (\phi)\right)} \cos (\phi) d \phi d \theta d \rho .
\end{aligned}
$$

We can break up the integral with respect to the variable $\phi$ which appears in $T_{1}$ and $T_{2}$ as

$$
\begin{aligned}
H(a, \rho, \theta) & =\int_{\theta}^{\theta+\delta} e^{-2 \pi i \frac{\rho}{a} \frac{1}{\kappa\left(1^{-}\right)} \cos (\phi)} \cos (\phi) d \phi \\
& =H_{1}(a, \rho)+H_{2}(a, \rho, \theta)+H_{3}(a, \rho, \theta),
\end{aligned}
$$

where

$$
\begin{aligned}
H_{1}(a, \rho) & =\int_{0}^{\delta} e^{-2 \pi i \frac{\rho}{a} \frac{1}{\kappa(1-)} \cos (\phi)} \cos (\phi) d \phi \\
H_{2}(a, \rho, \theta) & =\int_{\delta}^{\delta+\theta} e^{-2 \pi i \frac{\rho}{a} \frac{1}{\kappa\left(1^{-}\right)} \cos (\phi)} \cos (\phi) d \phi \\
H_{3}(a, \rho, \theta) & =-\int_{0}^{\theta} e^{-2 \pi i \frac{\rho}{a} \frac{1}{\kappa\left(1^{-}\right)} \cos (\phi)} \cos (\phi) d \phi .
\end{aligned}
$$

Hence, we can break up $T_{1}$ as $T_{1}=T_{11}+T_{12}+T_{13}$, where, for $j=1,2,3$, we have:

$$
\begin{aligned}
T_{1 j}(a, \infty, 0)= & \frac{a^{-\frac{1}{4}}}{2 \pi i \kappa\left(1^{-}\right)} \int_{0}^{\infty} \int_{-\pi / 2}^{\pi / 2} \hat{\psi}_{1}(\rho \cos \theta) \hat{\psi}_{2}\left(a^{-1 / 2} \tan \theta\right) e^{2 \pi i \frac{\rho}{a} \frac{1}{\kappa\left(1^{-}\right)} \cos \theta} \\
& \times H_{j}(a, \rho, \theta) d \theta d \rho .
\end{aligned}
$$

For the $T_{12}$ term, notice first that the support condition on $\hat{\psi}_{2}$ implies that $|\theta| \leq$ $a^{1 / 2}$; hence the integral $H_{2}$ is supported on an interval of size $|\theta| \leq a^{1 / 2}$. In addition, $(\cos \phi)^{\prime}=\sin \phi \neq 0$ for $\phi \in[\delta, \delta+\theta]$. Thus, one can integrate by parts the integral $H_{2}$ repeatedly. By doing this, one shows that the integral $T_{1 j}(a, \infty, 0)$ exhibits fast asymptotic decay as $a \rightarrow 0$.

To analyze $T_{13}$, we start applying the change of variable $\phi(\alpha)=a^{1 / 2} \alpha$ to the integral $H_{3}$ :

$$
H_{3}(a, \rho, \theta)=-\int_{0}^{a^{-1 / 2} \theta} e^{-2 \pi i \frac{\rho}{a} \frac{1}{\kappa\left(1^{-}\right)} \cos \left(a^{1 / 2} \alpha\right)} \cos \left(a^{1 / 2} \alpha\right) a^{1 / 2} d \alpha .
$$

Hence, the corresponding integral $T_{13}$ can be expressed as

$$
\begin{aligned}
T_{13}(a, \infty, 0)= & -\frac{a^{\frac{1}{4}}}{2 \pi i \kappa\left(1^{-}\right)} \int_{0}^{\infty} \int_{-\pi / 2}^{\pi / 2} \hat{\psi}_{1}(\rho \cos \theta) \hat{\psi}_{2}\left(a^{-1 / 2} \tan \theta\right) e^{2 \pi i \frac{\rho}{a} \frac{1}{\kappa\left(1^{-}\right)}(\cos \theta-1)} \\
& \times \int_{0}^{a^{-1 / 2} \theta} e^{-2 \pi i \frac{\rho}{a} \frac{1}{\kappa\left(1^{-}\right)}\left(\cos \left(a^{1 / 2} \alpha\right)-1\right)} \cos \left(a^{1 / 2} \alpha\right) d \alpha d \theta d \rho
\end{aligned}
$$


Using the change of variables $u=a^{-1 / 2} \tan \theta$ (from which: $\theta_{a}(u)=\arctan \left(a^{1 / 2} u\right.$ ), $\left.d \theta=\frac{a^{1 / 2} d u}{1+a u^{2}}, \cos \theta=\frac{1}{\sqrt{1+a u^{2}}}\right)$, we obtain:

$$
\begin{aligned}
T_{13}(a, \infty, 0)= & -\frac{a^{\frac{3}{4}}}{2 \pi i \kappa\left(1^{-}\right)} \int_{0}^{\infty} \int_{-1}^{1} \hat{\psi}_{1}\left(\rho \cos \theta_{a}(u)\right) \hat{\psi}_{2}(u) e^{2 \pi i \frac{\rho}{a} \frac{1}{\kappa\left(1^{-}\right)}\left(\frac{1}{\sqrt{1+a u^{2}}}-1\right)} \\
& \times \int_{0}^{a^{-1 / 2} \theta} e^{-2 \pi i \frac{\rho}{a} \frac{1}{\kappa\left(1^{-}\right)}\left(\cos \left(a^{1 / 2} \alpha\right)-1\right)} \cos \left(a^{1 / 2} \alpha\right) d \alpha \frac{d u}{1+a u^{2}} d \rho .
\end{aligned}
$$

Notice that $\lim _{a \rightarrow 0} a^{-1 / 2} \theta(u)=\lim _{a \rightarrow 0} a^{-1 / 2} \arctan \left(a^{1 / 2} u\right)=u, \lim _{a \rightarrow 0} \frac{\cos \left(a^{1 / 2} \alpha\right)-1}{a}=$ $-\frac{1}{2} \alpha^{2}$ and $\lim _{a \rightarrow 0} \frac{1-\frac{1}{\sqrt{1+a u^{2}}}}{a}=\frac{1}{2} u^{2}$. Hence:

$\lim _{a \rightarrow 0} a^{-3 / 4} T_{13}(a, \infty, 0)=-\frac{1}{2 \pi i \kappa\left(1^{-}\right)} \int_{0}^{\infty} \hat{\psi}_{1}(\rho) \int_{-1}^{1}\left(\int_{0}^{u} e^{\pi i \frac{\rho}{\kappa\left(1^{-}\right)} \alpha^{2}} d \alpha\right) \hat{\psi}_{2}(u) e^{-\pi i \frac{\rho}{\kappa(1-)} u^{2}} d u d \rho$.

Since $\int_{0}^{u} e^{\pi i \frac{\rho}{\kappa(1)} \alpha^{2}} d \alpha$ is an odd function for the variable $u$, and $\hat{\psi}_{2}$ is even, then the above quantity is 0 . This implies that $T_{13}(a, \infty, 0)$ is a $o\left(a^{3 / 4}\right)$.

Concerning $T_{11}$, notice first that

$$
\begin{aligned}
H_{1}(a, \rho) & =\int_{0}^{\delta} e^{-2 \pi i \frac{\rho}{a} \frac{1}{\kappa(1-)} \cos (\phi)} \cos (\phi) d \phi \\
& =\frac{1}{2} \int_{-\delta}^{\delta} e^{-2 \pi i \frac{\rho}{a} \frac{1}{\kappa(1-)} \cos (\phi)} \cos (\phi) d \phi .
\end{aligned}
$$

Let $G \in C_{0}^{\infty}(\mathbb{R})$ be a such that $G(t)=1$ for $|t| \leq \frac{1}{2} \delta$ and $G(t)=0$ for $|t|>\frac{3}{4} \delta$, and write

$$
\frac{1}{2} \int_{-\delta}^{\delta} e^{-2 \pi i \frac{\rho}{a} \frac{1}{\kappa\left(1^{-}\right)} \cos (\phi)} \cos (\phi) d \phi=J_{1}(a, \rho)+J_{2}(a, \rho)
$$

where

$$
\begin{aligned}
& J_{1}(a, \rho)=\frac{1}{2} \int_{-\delta}^{\delta} e^{-2 \pi i \frac{\rho}{a} \frac{1}{\kappa(1-)} \cos (\phi)} \cos (\phi) G(\phi) d \phi \\
& J_{2}(a, \rho)=\frac{1}{2} \int_{-\delta}^{\delta} e^{-2 \pi i \frac{\rho}{a} \frac{1}{\kappa\left(1^{-}\right)} \cos (\phi)} \cos (\phi)(1-G(\phi)) d \phi .
\end{aligned}
$$

By Lemma 4.1, it follows that $J_{2}(a, \rho)=O\left(a^{N}\right)$ as $a \rightarrow 0$ for any $N>0$. For $J_{1}(a, \rho)$, one can use Lemma.4.3 to show that

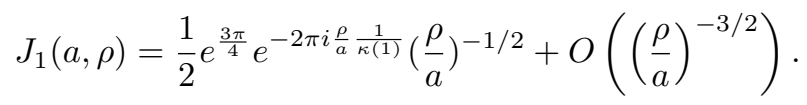

Thus, we may write $T_{11}$ (again omitting the higher order decay term) as

$T_{11}(a, \infty, 0)=\frac{a^{\frac{1}{4}} e^{\frac{3 \pi}{4} i}}{4 \pi i\left(\kappa\left(1^{-}\right)\right)^{\frac{1}{2}}} \int_{0}^{\infty} \int_{-\pi / 2}^{\pi / 2} \hat{\psi}_{1}(\rho \cos \theta) \hat{\psi}_{2}\left(a^{-1 / 2} \tan \theta\right) e^{2 \pi i \frac{\rho}{a} \frac{1}{\kappa(1)}(\cos \theta-1)} d \theta \rho^{-\frac{1}{2}} d \rho$

It follows from the argument for $T_{13}$ that

$$
\lim _{a \rightarrow 0} a^{-3 / 4} T_{11}(a, \infty, 0)=\frac{e^{\frac{3 \pi}{4} i}}{4 \pi i\left(\kappa\left(1^{-}\right)\right)^{\frac{1}{2}}} \int_{0}^{\infty} \hat{\psi}_{1}(\rho) \int_{-1}^{1} \hat{\psi}_{2}(u) e^{-\pi i \frac{\rho}{\kappa\left(1^{-}\right)} u^{2}} d u \rho^{-\frac{1}{2}} d \rho .
$$


Similarly one has

$$
\lim _{a \rightarrow 0} a^{-3 / 4} T_{21}(a, \infty, 0)=\frac{e^{\frac{\pi}{4} i}}{4 \pi i\left(\kappa\left(1^{-}\right)\right)^{\frac{1}{2}}} \int_{0}^{\infty} \hat{\psi}_{1}(\rho) \int_{-1}^{1} \hat{\psi}_{2}(u) e^{\pi i \frac{\rho}{\kappa(1-)} u^{2}} d u \rho^{-\frac{1}{2}} d \rho .
$$

Thus

$$
\begin{aligned}
& \lim _{a \rightarrow 0^{+}} a^{-\frac{3}{4}} \mathcal{S H}_{\psi} B(a, \infty, 0) \\
= & \lim _{a \rightarrow 0^{+}} a^{-\frac{3}{4}}\left(T_{11}(a, \infty, 0)+T_{21}(a, \infty, 0)\right) \\
= & \frac{1}{4 \pi i\left(\kappa\left(1^{-}\right)\right)^{\frac{1}{2}}} \int_{0}^{\infty} \hat{\psi}_{1}(\rho) \int_{-1}^{1} \hat{\psi}_{2}(u)\left[e^{\frac{3 \pi}{4} i} e^{-\pi i \frac{\rho}{\kappa\left(1^{-}\right)} u^{2}}+e^{\frac{\pi}{4} i} e^{\pi i \frac{\rho}{\kappa\left(1^{-}\right)} u^{2}}\right] d u \rho^{-\frac{1}{2}} d \rho \\
= & \frac{\sqrt{2}}{4 \pi\left(\kappa\left(1^{-}\right)\right)^{\frac{1}{2}}} \int_{0}^{\infty} \hat{\psi}_{1}(\rho) \int_{-1}^{1} \hat{\psi}_{2}(u)\left[\cos \left(\frac{\pi \rho}{\kappa\left(1^{-}\right)} u^{2}\right)+\sin \left(\frac{\pi \rho}{\left(\kappa\left(1^{-}\right)\right.} u^{2}\right)\right] d u \rho^{-\frac{1}{2}} d \rho \\
= & \left.\frac{\sqrt{2}}{4 \pi} \int_{0}^{\infty} \hat{\psi}_{1}\left(\kappa\left(1^{-}\right)\right) \rho\right) \int_{-1}^{1} \hat{\psi}_{2}(u)\left[\cos \left(\pi \rho u^{2}\right)+\sin \left(\pi \rho u^{2}\right)\right] d u \rho^{-\frac{1}{2}} d \rho
\end{aligned}
$$

Now it follows from Lemma 4.4 that

$$
\lim _{a \rightarrow 0} a^{-3 / 4} \mathcal{S H}_{\psi} B(a, \infty, 0)>0 .
$$

Case 2: $\kappa(1)=0$.

By Lemma 4.2, we may assume that $\mathcal{C}^{-}$is a segment of the tangent line of $\partial S$ at $(0,0)$. Thus, omitting faster asymptotic decay terms, we can write

$$
\begin{aligned}
& I_{1}^{-}(a, \infty, 0) \\
= & -\frac{a^{-\frac{1}{4}}}{2 \pi i} \int_{0}^{\infty} \int_{0}^{2 \pi} \hat{\psi}_{1}(\rho \sin \theta) \hat{\psi}_{2}\left(a^{-1 / 2} \cot \theta\right) \int_{-\epsilon}^{0} e^{-2 \pi i \frac{\rho}{a} \Theta(\theta) \cdot\left(x_{1}, 0\right)} \Theta(\theta) \cdot(0,-1) d x_{1} d \theta d \rho \\
= & -\frac{a^{-\frac{1}{4}}}{2 \pi i} \int_{0}^{\infty} \int_{0}^{2 \pi} \hat{\psi}_{1}(\rho \sin \theta) \hat{\psi}_{2}\left(a^{-1 / 2} \cot \theta\right) \int_{-\epsilon}^{0} e^{-2 \pi i \frac{\rho}{a} x_{1} \cos \theta} \sin \theta d x_{1} d \theta d \rho \\
= & \frac{a^{-\frac{1}{4}}}{2 \pi i} \int_{0}^{\infty} \int_{0}^{2 \pi} \hat{\psi}_{1}(\rho \cos \theta) \hat{\psi}_{2}\left(a^{-1 / 2} \tan \theta\right) \int_{-\epsilon}^{0} e^{2 \pi i \frac{\rho}{a} x_{1} \sin \theta} \cos \theta d x_{1} d \theta d \rho,
\end{aligned}
$$

where, in the last equation, we have used the change of variable $\theta \rightarrow \theta+\pi / 2$. Finally, similarly to Case 1 , we write $I_{1}^{-}(a, \infty, 0)=T_{1}(a, \infty, 0)+T_{2}(a, \infty, 0)$, where

$$
\begin{aligned}
& T_{1}(a, \infty, 0)=\frac{a^{-\frac{1}{4}}}{2 \pi i} \int_{0}^{\infty} \int_{-\pi / 2}^{\pi / 2} \hat{\psi}_{1}(\rho \cos \theta) \hat{\psi}_{2}\left(a^{-1 / 2} \tan \theta\right) \int_{-\epsilon}^{0} e^{2 \pi i \frac{\rho}{a} x_{1} \sin \theta} \cos \theta d x_{1} d \theta d \rho, \\
& T_{2}(a, \infty, 0)=\frac{a^{-\frac{1}{4}}}{2 \pi i} \int_{0}^{\infty} \int_{\pi / 2}^{3 \pi / 2} \hat{\psi}_{1}(\rho \cos \theta) \hat{\psi}_{2}\left(a^{-1 / 2} \tan \theta\right) \int_{-\epsilon}^{0} e^{2 \pi i \frac{\rho}{a} x_{1} \sin \theta} \cos \theta d x_{1} d \theta d \rho,
\end{aligned}
$$

To analyze $T_{1}(a, \infty, 0)$, it will be useful to introduce

$$
\widetilde{T}_{1}(a, \infty, 0)=\frac{a^{-\frac{1}{4}}}{2 \pi i} \int_{0}^{\infty} \int_{-\pi / 2}^{\pi / 2} \hat{\psi}_{1}(\rho) \hat{\psi}_{2}\left(a^{-1 / 2} \sin \theta\right) \int_{-\epsilon}^{0} e^{2 \pi i \frac{\rho}{a} x_{1} \sin \theta} \cos \theta d x_{1} d \theta d \rho
$$


For $|\theta| \leq C a^{1 / 2}$, we have

$$
\begin{aligned}
& \left|\hat{\psi}_{1}(\rho \cos \theta) \hat{\psi}_{2}\left(a^{-1 / 2} \tan \theta\right)-\hat{\psi}_{1}(\rho) \hat{\psi}_{2}\left(a^{-1 / 2} \sin \theta\right)\right| \\
& \leq\left|\hat{\psi}_{1}(\rho \cos \theta)-\hat{\psi}_{1}(\rho)\right|\left|\hat{\psi}_{2}\left(a^{-1 / 2} \tan \theta\right)\right|+\left|\hat{\psi}_{1}(\rho)\right|\left|\hat{\psi}_{2}\left(a^{-1 / 2} \tan \theta\right)-\hat{\psi}_{2}\left(a^{-1 / 2} \sin \theta\right)\right| \\
& \leq C\left(|\cos \theta-1|+a^{-1 / 2}|\tan \theta-\sin \theta|\right) \\
& \leq C\left(\theta^{2}+a^{-1 / 2}|\tan \theta||1-\cos \theta|\right) \\
& \leq C\left(\theta^{2}+a^{-1 / 2}|\theta| \theta^{2}\right) \leq C a .
\end{aligned}
$$

Hence, noticing that the integration with respect to $\theta$ yields a term $C a^{1 / 2}$, we obtain

$$
\left|T_{1}(a, \infty, 0)-\widetilde{T}_{1}(a, \infty, 0)\right| \leq C a^{-1 / 4} a^{1 / 2} a=C a^{5 / 4} .
$$

Using the change of variables $u(\theta)=a^{-1 / 2} \sin \theta$, we have:

$$
\begin{aligned}
\widetilde{T}_{1}(a, \infty, 0) & =\frac{a^{\frac{1}{4}}}{2 \pi i} \int_{0}^{\infty} \int_{-1}^{1} \hat{\psi}_{1}(\rho) \hat{\psi}_{2}(u) \int_{-\epsilon}^{0} e^{2 \pi i \frac{\rho}{\sqrt{a}} u x_{1}} d x_{1} d u d \rho \\
& =\frac{a^{\frac{1}{4}}}{2 \pi i} \int_{0}^{\infty} \hat{\psi}_{1}(\rho) \int_{-\epsilon}^{0} \int_{-1}^{1} \hat{\psi}_{2}(u) e^{2 \pi i \frac{\rho}{\sqrt{a}} u x_{1}} d u d x_{1} d \rho \\
& =\frac{a^{\frac{1}{4}}}{2 \pi i} \int_{0}^{\infty} \hat{\psi}_{1}(\rho) \int_{-\epsilon}^{0} \psi_{2}\left(\frac{\rho x_{1}}{\sqrt{a}}\right) d x_{1} d \rho \\
& =\frac{a^{\frac{3}{4}}}{2 \pi i} \int_{0}^{\infty} \hat{\psi}_{1}(\rho) \int_{-\frac{\rho \epsilon}{\sqrt{a}}}^{0} \psi_{2}(x) d x \frac{d \rho}{\rho},
\end{aligned}
$$

where, in the last equality, we have used the change of variable $x=\frac{\rho}{\sqrt{a}} x_{1}$. Hence

$$
\lim _{a \rightarrow 0} a^{-\frac{3}{4}} T_{1}(a, \infty, 0)=(2 \pi i)^{-1} \int_{0}^{\infty} \hat{\psi}_{1}(\rho) \frac{d \rho}{\rho} \int_{-\infty}^{0} \psi_{2}(x) d x
$$

Similarly one has

$$
\lim _{a \rightarrow 0} a^{-\frac{3}{4}} T_{2}(a, \infty, 0)=(2 \pi i)^{-1} \int_{0}^{\infty} \hat{\psi}_{1}(\rho) \frac{d \rho}{\rho} \int_{0}^{\infty} \psi_{2}(x) d x .
$$

Thus

$$
\begin{aligned}
\lim _{a \rightarrow 0} a^{-3 / 4} \mathcal{S H}_{\psi} B(a, \infty, 0) & =(2 \pi i)^{-1} \int_{0}^{\infty} \hat{\psi}_{1}(\rho) \frac{d \rho}{\rho} \int_{-\infty}^{\infty} \psi_{2}(x) d x \\
& =(2 \pi i)^{-1} \hat{\psi}_{2}(0) \int_{0}^{\infty} \hat{\psi}_{1}(\rho) \frac{d \rho}{\rho} \neq 0 .
\end{aligned}
$$

This finishes the proof of Theorem 3.2.

5.2. Proof of Theorem 3.1. (Part (i)) Since $p=(0,0)$ is a regular point, there is no need to split $C$ as $C^{-}$and $C^{+}$. Thus, as in the proof of (i) of Theorem 3.2, we let

$$
I_{1}\left(a, s_{0}, 0\right)=-\frac{a^{-\frac{1}{4}}}{2 \pi i} \int_{0}^{\infty} \int_{0}^{2 \pi} \hat{\psi}_{1}(\rho \cos \theta) \hat{\psi}_{2}\left(a^{-1 / 2}\left(\tan \theta-\tan \theta_{0}\right)\right) K(a, \rho, \theta) d \theta d \rho
$$


where

$$
K(a, \rho, \theta)=\int_{1-\epsilon}^{1+\epsilon} e^{-2 \pi i \frac{\rho}{a} \Theta(\theta) \cdot \vec{\alpha}(t)} \Theta(\theta) \cdot n(t) d t .
$$

Let $G(t) \in C_{0}^{\infty}(\mathbb{R})$ with $G(t)=1$ for $|t-1| \leq \frac{\epsilon}{4}$ and $G(t)=0$ for $|t-1|>\frac{3 \epsilon}{4}$. Let

$$
\begin{aligned}
K_{1}(a, \rho, \theta) & =\int_{1-\epsilon}^{1+\epsilon} e^{-2 \pi i \frac{\rho}{a} \Theta(\theta) \cdot \vec{\alpha}(t)} \Theta(\theta) \cdot n(t) G(t) d t \\
K_{2}(a, \rho, \theta) & =\int_{1-\epsilon}^{1+\epsilon} e^{-2 \pi i \frac{\rho}{a} \Theta(\theta) \cdot \vec{\alpha}(t)} \Theta(\theta) \cdot n(t)(1-G(t)) d t \\
I_{11}\left(a, s_{0}, 0\right) & =-\frac{a^{-\frac{1}{4}}}{2 \pi i} \int_{0}^{\infty} \int_{0}^{2 \pi} \hat{\psi}_{1}(\rho \cos \theta) \hat{\psi}_{2}\left(a^{-1 / 2}\left(\tan \theta-\tan \theta_{0}\right)\right) K_{1}(a, \rho, \theta) d \theta d \rho, \\
I_{12}\left(a, s_{0}, 0\right) & =-\frac{a^{-\frac{1}{4}}}{2 \pi i} \int_{0}^{\infty} \int_{0}^{2 \pi} \hat{\psi}_{1}(\rho \cos \theta) \hat{\psi}_{2}\left(a^{-1 / 2}\left(\tan \theta-\tan \theta_{0}\right)\right) K_{2}(a, \rho, \theta) d \theta d \rho,
\end{aligned}
$$

such that $I_{1}\left(a, s_{0}, 0\right)=I_{11}\left(a, s_{0}, 0\right)+I_{12}\left(a, s_{0}, 0\right)$.

Applying Lemma 4.1 to $I_{12}\left(a, s_{0}, 0\right)$, it follows that $\left|I_{12}\left(a, s_{0}, 0\right)\right| \leq C_{N} a^{N}$ for any $N>0$. Since $s_{0}$ does not correspond to the normal direction at $p$, we see that $\Theta(\theta) \cdot \vec{\alpha}^{\prime}(t) \neq 0$ for $t$ near 1 and $\theta$ near $\theta_{0}$. It follows from repeated integration by parts that $\left|I_{11}\left(a, s_{0}, 0\right)\right| \leq C_{N} a^{N}$.

Part (ii)) This can be proved by following the argument for (iii) of Theorem 3.2 with $C^{-}$replaced by $C$.

This finishes the proof of Theorem 3.1.

Finally, notice that the proof of Proposition 3.3 follows easily from a closer examination of the arguments of parts (i) and (ii) of Theorem 3.2.

6. Algorithms for edge analysis. To demonstrate the potential of the shearlet framework in the analysis of edges, we will now briefly illustrate how the properties of the shearlet transform can be exploited to identify smooth regions and edges, and to distinguish regular edge points from different kind of junctions and corner points. Let us consider the simple image $u$, in Figure 6.1(a), containing edges and smooth regions. Figure 6.1(b) shows several plots of the discrete shearlet transform $\mathcal{S H} u[j, \ell, k]$, at a fixed scale 'fine' $j_{0}$, for some representative locations $k_{0}$. Notice that the discrete shearlet transform maps the image $u$ into coefficients depending on the discrete scales $2^{-j}, j \in \mathbb{Z}$, orientations $1 \leq \ell \leq 2^{j+1}$, and locations $k \in \mathbb{Z}^{2}$. We refer to $[5,16]$ for details about the discrete shearlet transform and its numerical implementation. As Figure 6.1 indicates, we can recognize four classes of points from the plot of $S_{k_{0}}(\ell)=\left|\mathcal{S H} u\left[j_{0}, \ell, k_{0}\right]\right|$. Namely, at the junction point $k_{0}=A, S_{A}(\ell)$ exhibit three peaks corresponding to the three normal orientations to the three edge segments converging into $A$; at the point $k_{0}=B$ on a smooth edge, $S_{B}(\ell)$ has a single peak; at a point $k=C$ in a smooth region, $S_{C}(\ell)$ is essentially flat; finally, at a point $k_{0}=C$ "close" to an edge, $S_{D}(\ell)$ exhibits two peaks but they are much smaller in amplitude than those for the points $A$ and $B$. The same behavior holds, essentially, for more general images. In particular, in the presence of a corner point, the behavior is similar to point $A$, with the plot of the shearlet transform showing two main peaks corresponding to the orientations of the two normal orientations at the corner point.

Based on these observations, one can design simple algorithms for classifying regular edge points, corner points and different kind of junctions points based on 
their geometrical properties. We refer to $[15,16]$ for additional details about this and other algorithms for edge analysis and detection, and for extensive numerical demonstrations.

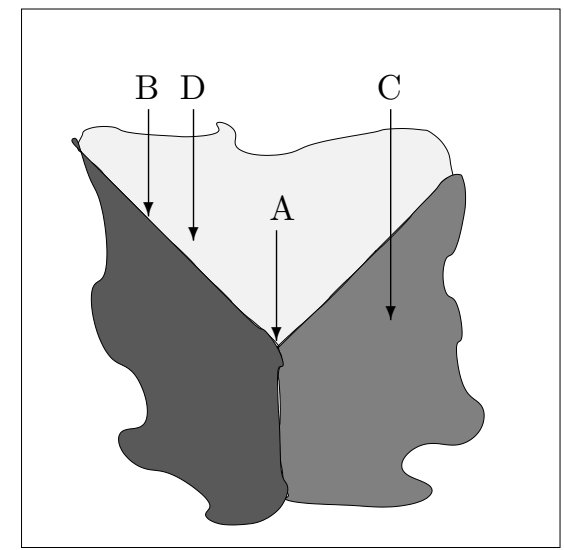

(a)
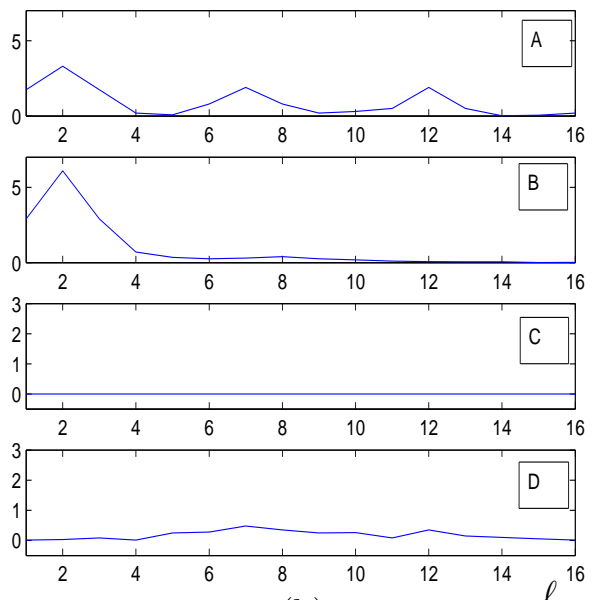

(b)

FIG. 6.1. (a) Test image and representative points $A$ (junction), $B$ (regular edge point), $C$ (smooth region), $D$ (near edge). (b) Magnitude of the Discrete Shearlet Transform $S_{k}(\ell)$, as a function of the orientation parameter $\ell$ for the points $k$ indicated in (a). Notice the different scaling factor used in the $y$-axis, for the plots of points $C$ and $D$.

\section{Appendix A. Proof of Lemma 4.4.}

Here is the proof of Lemma 4.4.

Proof. Let $g(\rho)=\int_{0}^{1} \hat{\psi}_{2}(u)\left(\sin \left(\pi \rho u^{2}\right)+\cos \left(\pi \rho u^{2}\right)\right) d u$. By a change of variable, we obtain:

$$
g(\rho)=\int_{0}^{\rho} v^{-\frac{1}{2}} \hat{\psi}_{2}\left(\sqrt{\frac{v}{\rho}}\right)(\sin (\pi v)+\cos (\pi v)) d v=g_{1}(\rho)+g_{2}(\rho),
$$

where

$$
\begin{aligned}
& g_{1}(\rho)=\int_{0}^{\rho} v^{-\frac{1}{2}} \hat{\psi}_{2}\left(\sqrt{\frac{v}{\rho}}\right) \sin (\pi v) d v \\
& g_{2}(\rho)=\int_{0}^{\rho} v^{-\frac{1}{2}} \hat{\psi}_{2}\left(\sqrt{\frac{v}{\rho}}\right) \cos (\pi v) d v .
\end{aligned}
$$

If $\rho \leq 1$, it is trivial to see that $g_{1}(\rho)>0$ since $\hat{\psi}_{2}(0) \geq \frac{1}{2}, \hat{\psi}_{2}(x) \geq 0$ on $[0,1]$ and $\sin (\pi x)>0$ on $(0,1)$. Now assume that $1<\rho \leq 2$. Since $\hat{\psi}_{2}(x)$ is decreasing on $(0,1)$, we have

$$
\begin{aligned}
g_{1}(\rho)= & \int_{0}^{1} v^{-\frac{1}{2}} \hat{\psi}_{2}\left(\sqrt{\frac{v}{\rho}}\right) \sin (\pi v) d v+\int_{1}^{\rho} v^{-\frac{1}{2}} \hat{\psi}_{2}\left(\sqrt{\frac{v}{\rho}}\right) \sin (\pi v) d v \\
& =\int_{0}^{1} v^{-\frac{1}{2}} \hat{\psi}_{2}\left(\sqrt{\frac{v}{\rho}}\right) \sin (\pi v) d v-\int_{0}^{\rho-1}(v+1)^{-\frac{1}{2}} \hat{\psi}_{2}\left(\sqrt{\frac{v+1}{\rho}}\right) \sin (\pi v) d v \\
& \geq \int_{0}^{1}\left(v^{-\frac{1}{2}} \hat{\psi}_{2}\left(\sqrt{\frac{v}{\rho}}\right)-(v+1)^{-\frac{1}{2}} \hat{\psi}_{2}\left(\sqrt{\frac{v+1}{\rho}}\right)\right) \sin (\pi v) d v>0 .
\end{aligned}
$$


For $\rho>2$, one can find $k \geq 1$ and $0<\zeta \leq 2$ such that $\rho=2 k+\zeta$. In this case, we have:

$$
\begin{aligned}
g_{1}(\rho) & =\int_{0}^{2 k} v^{-\frac{1}{2}} \hat{\psi}_{2}\left(\sqrt{\frac{v}{\rho}}\right) \sin (\pi v) d v+\int_{2 k}^{\rho} v^{-\frac{1}{2}} \hat{\psi}_{2}\left(\sqrt{\frac{v}{\rho}}\right) \sin (\pi v) d v \\
& =g_{0}(\rho)+g_{\zeta}(\rho),
\end{aligned}
$$

where

$$
\begin{aligned}
g_{0}(\rho) & =\sum_{j=0}^{k-1} \int_{0}^{1}\left((v+2 j)^{-\frac{1}{2}} \hat{\psi}_{2}\left(\sqrt{\frac{v+2 j}{\rho}}\right)+\right. \\
& \left.-(v+2 j+1)^{-\frac{1}{2}} \hat{\psi}_{2}\left(\sqrt{\frac{v+2 j+1}{\rho}}\right)\right) \sin (\pi v) d v ; \\
g_{\zeta}(\rho) & =\int_{0}^{\zeta}(v+2 k)^{-\frac{1}{2}} \hat{\psi}_{2}\left(\sqrt{\frac{v+2 k}{\rho}}\right) \sin (\pi v) d v .
\end{aligned}
$$

It is easy to verify that $g_{0}>0$ and $g_{\zeta} \geq 0$ and hence $g_{1}>0$.

Next we consider $g_{2}(\rho)$. If $\rho \leq \frac{1}{2}$, it is trivial to see that $g_{2}(\rho)>0$ since $\cos (\pi x)>$ 0 on $\left(0, \frac{1}{2}\right)$. Now assume that $\frac{1}{2}<\rho \leq \frac{3}{2}$. Since $\cos (\pi v)<0$ on $\left(\frac{1}{2}, \frac{3}{2}\right)$, we have

$$
\begin{aligned}
g_{2}(\rho) & =\int_{0}^{\frac{1}{2}} v^{-\frac{1}{2}} \hat{\psi}_{2}\left(\sqrt{\frac{v}{\rho}}\right) \cos (\pi v) d v+\int_{\frac{1}{2}}^{\rho} v^{-\frac{1}{2}} \hat{\psi}_{2}\left(\sqrt{\frac{v}{\rho}}\right) \cos (\pi v) d v \\
& \geq \int_{0}^{\frac{1}{2}} v^{-\frac{1}{2}} \hat{\psi}_{2}\left(\sqrt{\frac{v}{\rho}}\right) \cos (\pi v) d v+\int_{0}^{\frac{3}{2}} v^{-\frac{1}{2}} \hat{\psi}_{2}\left(\sqrt{\frac{v}{\rho}}\right) \cos (\pi v) d v \\
& \geq c_{0} \hat{\psi}_{2}\left(\sqrt{\frac{1}{2 \rho}}\right)
\end{aligned}
$$

where $c_{0}=\int_{0}^{\frac{3}{2}} v^{-\frac{1}{2}} \cos (\pi v) d v$. Since $\int_{0}^{\sqrt{\frac{3 \pi}{2}}} \cos u^{2} d u \geq 0.4>0$, it follows that $c_{0}>0$.

For $\frac{3}{2}<\rho \leq \frac{5}{2}$, since $\cos (\pi v)>0$ on $\left(\frac{3}{2}, \frac{5}{2}\right)$, we have:

$$
\begin{aligned}
g_{2}(\rho) & =\int_{0}^{\frac{1}{2}} v^{-\frac{1}{2}} \hat{\psi}_{2}\left(\sqrt{\frac{v}{\rho}}\right) \cos (\pi v) d v+\int_{\frac{1}{2}}^{\frac{3}{2}} v^{-\frac{1}{2}} \hat{\psi}_{2}\left(\sqrt{\frac{v}{\rho}}\right) \cos (\pi v) d v \\
& +\int_{\frac{3}{2}}^{\rho} v^{-\frac{1}{2}} \hat{\psi}_{2}\left(\sqrt{\frac{v}{\rho}}\right) \cos (\pi v) d v \\
& \geq \int_{0}^{\frac{1}{2}} v^{-\frac{1}{2}} \hat{\psi}_{2}\left(\sqrt{\frac{v}{\rho}}\right) \cos (\pi v) d v+\int_{\frac{1}{2}}^{\frac{3}{2}} v^{-\frac{1}{2}} \hat{\psi}_{2}\left(\sqrt{\frac{v}{\rho}}\right) \cos (\pi v) d v \\
& \geq c_{0} \hat{\psi}_{2}\left(\sqrt{\frac{1}{2 \rho}}\right)>0 .
\end{aligned}
$$

For $\rho>\frac{5}{2}$, one can find $n \geq 1$ and $0 \leq \zeta<1$ such that $\rho=\frac{3}{2}+n+\zeta$. Let us examine the cases where $n$ is even and odd separately. If $n=2 k$, for some $k \geq 1$, we 
have

$$
\begin{aligned}
& g_{2}(\rho) \\
\geq & \hat{\psi}_{2}\left(\sqrt{\frac{1}{2 \rho R}}\right) \int_{0}^{\frac{3}{2}} v^{-\frac{1}{2}} \cos (\pi v) d v \\
+ & \sum_{j=0}^{k-1} \int_{\frac{3}{2}}^{\frac{5}{2}}\left((v+2 j)^{-\frac{1}{2}} \hat{\psi}_{2}\left(\sqrt{\frac{v+2 j}{\rho}}\right)-(v+2 j+1)^{-\frac{1}{2}} \hat{\psi}_{2}\left(\sqrt{\frac{v+2 j+1}{\rho}}\right)\right) \cos (\pi v) d v \\
+ & \int_{\frac{3}{2}}^{\frac{3}{2}+\zeta}(v+2 k)^{-\frac{1}{2}} \hat{\psi}_{2}\left(\sqrt{\frac{v+2 k}{\rho}}\right) \cos (\pi v) d v \\
\geq & c_{0} \hat{\psi}_{2}\left(\sqrt{\frac{1}{2 \rho}}\right)>0 .
\end{aligned}
$$

If $n=2 k+1$, for some $k \geq 1$, we have

$$
\begin{aligned}
& g_{2}(\rho) \\
\geq & \hat{\psi}_{2}\left(\sqrt{\frac{1}{2 \rho}}\right) \int_{0}^{\frac{3}{2}} v^{-\frac{1}{2}} \cos (\pi v) d v \\
+ & \sum_{j=0}^{k-1} \int_{\frac{3}{2}}^{\frac{5}{2}}\left((v+2 j)^{-\frac{1}{2}} \hat{\psi}_{2}\left(\sqrt{\frac{v+2 j}{\rho}}\right)-(v+2 j+1)^{-\frac{1}{2}} \hat{\psi}_{2}\left(\sqrt{\frac{v+2 j+1}{\rho R}}\right)\right) \cos (\pi v) d v \\
+ & \int_{\frac{3}{2}}^{\frac{5}{2}}(v+2 k)^{-\frac{1}{2}} \hat{\psi}_{2}\left(\sqrt{\frac{v+2 k}{\rho}}\right) \cos (\pi v) d v+ \\
- & \int_{\frac{3}{2}}^{\frac{3}{2}+\zeta}(v+2 k+1)^{-\frac{1}{2}} \hat{\psi}_{2}\left(\sqrt{\frac{v+2 k+1}{\rho}}\right) \cos (\pi v) d v \\
\geq & c_{0} \hat{\psi}_{2}\left(\sqrt{\frac{1}{2 \rho}}\right)>0 .
\end{aligned}
$$

This completes the proof.

Acknowledgments. The authors thank Glenn Easley for valuable discussions and Sheng Yi for providing the data for Figure 6.1. DL acknowledges support from NSF grant DMS 0604561 and DMS (Career) 0746778.

\section{REFERENCES}

[1] E. J. Candès and D. L. Donoho, New tight frames of curvelets and optimal representations of objects with $C^{2}$ singularities, Comm. Pure Appl. Math. 56 (2004), 219-266.

[2] E. J. Candès and D. L. Donoho, Continuous curvelet transform: I. Resolution of the wavefront set, Appl. Comput. Harmon. Anal. 19 (2005), 162-197.

[3] T. Chan and J. Shen, Image Processing and Analysis, SIAM, Philadelphia, 2005.

[4] M. Do Carmo, Differential geometry of Curves and Surfaces, Prentice Hall, 1976.

[5] G. Easley, D. Labate, and W-Q. Lim Sparse Directional Image Representations using the Discrete Shearlet Transform, to appear in Appl. Computat. Harmon. Anal., 2008.

[6] K. Guo, G. Kutyniok, and D. Labate, Sparse multidimensional representations using anisotropic dilation and shear operators, in: Wavelets and Splines, G. Chen and M. Lai (eds.), Nashboro Press, Nashville, TN (2006), 189-201.

[7] K. Guo and D. Labate, Optimally sparse multidimensional representation using shearlets, SIAM J. Math. Anal., 39 (2007), 298-318.

[8] K. Guo, W. Lim, D. Labate, Edge analysis using the continuous shearlet transform, to appear in Appl. Computat. Harmon. Anal. (2008). 
[9] C.S. Herz, Fourier transforms related to convex sets, Ann. of Math. 75 (1962), 81-92.

[10] M. Holschneider, Wavelets. Analysis tool, Oxford University Press, Oxford, 1995.

[11] G. Kutyniok and D. Labate, Resolution of the Wavefront Set using Continuous Shearlets, to appear in Trans AMS.

[12] D. G. Lowe, Distinctive Image Features from Scale-Invariant Keypoints, Int. J. Comp. Vision 60 (2004), 91-110.

[13] Y. Meyer, Wavelets and Operators, Cambridge Stud. Adv. Math. vol. 37, Cambridge Univ. Press, Cambridge, UK, 1992.

[14] E. M. Stein, Harmonic Analysis: real-variable methods, orthogonality, and oscillatory integrals, Princeton University Press, Princeton, NJ, 1993.

[15] S. Yi, D. Labate, G. R. Easley, and H. Krim, Edge Detection and Processing using Shearlets, to appear in IEEE Int. Conf. on Image Processing (ICIP), (2008).

[16] S. Yi, D. Labate, G. R. Easley, and H. Krim, A Shearlet Approach to Edge Analysis and Detection, preprint (2008) (available at http://www4.ncsu.edu/ dlabate/publications.html)

[17] D. Ziou, and S. Tabbone, Edge Detection Techniques An Overview, International Journal of Pattern Recognition and Image Analysis 8(4) (1998), 537-559. 